Mon. Not. R. Astron. Soc. 000, 1-17 (0000) Printed 26 March $2020 \quad$ (MN IaT $\mathrm{T}_{\mathrm{E}} \mathrm{X}$ style file v2.2)

\title{
The Population of Galaxies that Contribute to The HI Mass Function
}

\author{
Saili Dutta ${ }^{1 \star}$, Nishikanta Khandai ${ }^{1 \star}$, Biprateep Dey ${ }^{1,2 \star}$ \\ 1 School of Physical Sciences, National Institute of Science Education and Research, HBNI, Jatni 752050, India \\ 2 Pittsburgh Particle Physics, Astrophysics and Cosmology Center (PITT PACC) and Department of Physics and Astronomy, \\ University of Pittsburgh, 3941 O'Hara Street, Pittsburgh, PA 15260, USA
}

26 March 2020

\begin{abstract}
We look at the contribution of different galaxy populations to the atomic hydrogen (HI) mass function (HIMF) and the HI density parameter, $\Omega_{\mathrm{HI}}$, in the local Universe. Our analysis is based on a sample of $7857 \mathrm{HI}$-selected galaxies selected from a volume common to the SDSS and ALFALFA (40\% catalog - $\alpha .40)$ surveys. We define different populations of galaxies in the color $(u-r)$-magnitude $\left(M_{\mathrm{r}}\right)$ plane and compute the HIMF for each of them. Additionally we compute the HIMF for dark galaxies; these are undetected in SDSS and represent $\sim 2 \%$ of the total sample. We find that the luminous red population dominates the total HIMF for $\log _{10}\left(M_{\mathrm{HI}} h_{70}^{2} / M_{\odot}\right) \geq 10.4$. The full red population - luminous and faint - represents about $\sim 17 \%$ of the $\Omega_{\mathrm{HI}}$ budget, while that of the dark population is $\sim 3 \%$. The HIMF about the knee, $\log _{10}\left(M_{\mathrm{HI}} h_{70}^{2} / M_{\odot}\right) \in[8,10.4]$, is dominated by the faint and luminous blue populations, the latter dominating at larger masses in this interval. Their total contribution to $\Omega_{\mathrm{HI}}$ is $\sim 55-70 \%$, the variation depending on the definition of population. The dominant populations at the low mass end, $\log _{10}\left(M_{\mathrm{HI}} h_{70}^{2} / M_{\odot}\right) \leq 8.0$ are the faint blue and faint bluer populations, the latter's dominance being sensitive to its definition. The full blue (blue-bluer luminous and faint) population represents $\sim 80 \%$ of $\Omega_{\mathrm{HI}}$. A bimodal HIMF suggested by our results is however not seen since the amplitude of the HIMF of the luminous red population is small compared to that of the luminous blue population.
\end{abstract}

Key words: galaxies: formation - galaxies: evolution - galaxies: luminosity function, mass function - radio lines: galaxies - surveys

\section{INTRODUCTION}

The relationship between gas, metals, feedback and stars in galaxies is crucial for our understanding of galaxy formation and evolution. We need a clearer picture of how dark matter halos, which harbor galaxies, are supplied with cold gas, the fuel for star formation; how local and global conditions in the galaxy are responsible in processing them into stars; and finally how the gas is polluted with metals and recycled back to the intergalactic medium due to feedback processes within the galaxy.

Three basic properties that describe galaxies are: (i) the star formation rate (SFR), (ii) the stellar mass $\left(M_{\text {star }}\right)$ and (iii) the cold neutral hydrogen gas mass both in molecular $\left(M_{\mathrm{H}_{2}}\right)$ and atomic $\left(M_{\mathrm{HI}}\right)$ phases. The amount of neutral gas

\footnotetext{
* E-mail: sailidutta@niser.ac.in(SD); nkhandai@niser.ac.in(NK); biprateep@pitt.edu(BD)
}

tells us the amount of fuel currently available for future star formation. The SFR is the current rate of forming stars from the supply of cold gas that is available, while $M_{\text {star }}$ depends on the integrated star formation history. Within each galaxy, at sub-kpc scales, the observed correlation between surface density of molecular hydrogen, $\Sigma_{\mathrm{H}_{2}}$, and the SFR surface density, $\Sigma_{\mathrm{SFR}}$, is stronger (Bigiel, et al. 2008; Leroy, et al. 2008) as compared to the correlation between the HI surface density, $\Sigma_{\mathrm{HI}}$, and $\Sigma_{\mathrm{SFR}}$. The HI is often distributed beyond the optical radius of the galaxy and is more diffuse whereas star formation and $\mathrm{H}_{2}$ are mostly concentrated within the optical radius and occur in clumpier regions (Leroy, et al. 2008). However when the HI gas cools and becomes denser it transitions to molecular gas, which cools further and becomes denser leading eventually to gravitational collapse to form stars. It is therefore common to correlate the total gas $\left(\mathrm{HI}+\mathrm{H}_{2}\right)$ to the SFR, the so-called Kennicutt-Schmidt law 
(Schmidt 1959, 1963; Kennicutt 1998, 1989) for star formation, where $\Sigma_{\mathrm{SFR}} \propto \Sigma_{\text {gas }}^{1.4}$.

With the aid of multiwavelength observations in the optical, ultraviolet (UV) and infrared (IR) bands followed up by spectroscopic measurements, we are able to infer scaling relations between various properties of galaxies, derived and/or observed. These scaling relations are very important since any theoretical model of galaxy formation should be able to reproduce them. In practice, these relations are used as parameters in theoretical models of galaxy formation when studied in cosmological volumes, since the physics on small, subparsec scales is never resolved (Springel \& Hernquist 2003; Di Matteo, Springel \& Hernquist 2005). A multipronged approach is used to study the distribution of $\mathrm{HI}$ in the post reionization Universe; these include cosmological hydrodynamical simulations of galaxy formation (Davé, et al. 2017), semi-analytical models (SAM) of galaxy formation (Kim, et al. 2017) and halo-occupation distribution (HOD) (Paul, Choudhury \& Paranjape 2018). All of these approaches invoke in some form or other some of the observed scaling relations and model predictions are tested against observations which were not used as their input. Since these scaling relations depend on the observed sample they may be biased, i.e. the relations may depend on how the sample is chosen. E.g. the $M_{\mathrm{HI}}-M_{\text {star }}$ relation differs if a sample is chosen by HI mass (Huang, et al. 2012) or stellar mass (Catinella, et al. 2010). On the other hand quantities like the luminosity function, mass function, correlation function (to name a few) are corrected for the survey selection and tell us about the underlying abundance and distribution of different galaxy types in the survey volume.

By analyzing data from optical, UV and IR surveys over the past decade we have formed a clearer picture of how galaxies, on average, have formed and evolved over the past $\sim 12.5$ billion years from redshift $z=6$ to today. The cosmic stellar mass density, $\rho_{*}$ (units of $M_{\odot} \mathrm{Mpc}^{-3} 3$ ), has increased monotonically by nearly 2.5 decades from $\log \left(\rho_{*}\right) \simeq 6.3$ at $z=6$ to $\log \left(\rho_{*}\right) \simeq 8.8$ today (Madau \& Dickinson 2014). These observations also tell us how the cosmic SFR density (SFRD denoted by $\psi$ with units $M_{\odot} \mathrm{yr}^{-1} \mathrm{Mpc}^{-3}$ ) has changed during this time. It increases from $\log (\psi)=-1.7$ at $z=6$ to a peak value of $\log (\psi)=-0.9$ at $z \simeq 2$ and finally dropping by a decade to $\log (\psi)=-1.8$ today (Le Floc'h, et al. 2005; Madau \& Dickinson 2014; Casey, Narayanan \& Cooray 2014). In contrast, surveys targeting gas content of galaxies have lagged behind in depth and number. The $21 \mathrm{~cm}$ line of HI being a weak line its detection in emission is limited only to the local Universe.

Blind HI surveys like the HI Parkes All Sky Survey (HIPASS, Meyer, et al. 2004) and the Arecibo Fast Legacy ALFA survey (ALFALFA, Giovanelli, et al. 2005) have been used to accurately measure the HIMF (Zwaan, et al. 2003, 2005; Martin, et al. 2010; Haynes, et al. 2011; Jones, et al. $2018)$ in the local Universe $(z \leq 0.05)$. The HIMF can then be integrated to obtain the $\mathrm{HI}$ density parameter $\Omega_{\mathrm{HI}}$. At higher redshifts the $21 \mathrm{~cm}$ flux gets further diluted and direct detection becomes difficult with existing instruments. Stacking the HI data on known optical counterparts or alternately cross-correlating the HI intensity maps with the optical catalog in a common volume, then becomes a useful tool in making detections. The stacking method has been applied for star-forming galaxies at $z=0.24$ (Lah, et al.
2007), galaxies in a cluster environment at $z=0.37$ (Lah, et al. 2009), for field galaxies at $z=0.1-0.2$ (Rhee, et al. 2013), in the $\mathrm{zCOSMOS}^{1}$ field at $z=0.37$ (Rhee, et al. 2016) and the $\mathrm{VVDS}^{2}$ field at $z=0.32$ (Rhee, et al. 2018). Most of the HI observations using the stacking method were done with the Giant Metrewave Radio Telescope (GMRT). More recently stacking has been applied to estimate the HI content in filaments (Tramonte, et al. 2019). However at higher redshifts $(z \sim 1.3)$ the stacking method did not result in a detection of star forming galaxies in the DEEP2 ${ }^{3}$ field (Kanekar, Sethi \& Dwarakanath 2016) whereas positive detections have been reported by cross-correlating the HI Intensity map with an optical survey at $z \sim 0.8$ (Chang, et al. 2010; Masui, et al. 2013). In the stacking method one can estimate $\Omega_{\mathrm{HI}}$ after correcting for the optical survey's completeness limit, whereas the cross-correlation method constrains $\Omega_{\mathrm{HI}} b_{\mathrm{HI}} r$. Here $b_{\mathrm{HI}}$ and $r$ are the HI bias parameter and galaxy-HI cross correlation coefficient. In summary these HI surveys constrain $\Omega_{\mathrm{HI}}=4 \pm 1.6 \times 10^{-4}$ out to $z \simeq 0.4$ (Rhee, et al. 2018).

Beyond $z=0.4$ and out to $z \simeq 5$ the HI content of the Universe is derived from studying damped Lyman- $\alpha$ systems (DLA) seen, in absorption, in the spectra of quasars (Prochaska, Herbert-Fort \& Wolfe 2005; Noterdaeme, et al. 2012; Neeleman, et al. 2016; Rao, et al. 2017). Combining both these approaches at low redshift (emission) and high redshift (absorption) one sees that $\Omega_{\mathrm{HI}}$ increases monotonically as $(1+z)^{0.56}$ from $\Omega_{\mathrm{HI}}(z=0)=4 \times 10^{-4}$ to $\Omega_{\mathrm{HI}}(z=5)=1.1 \times 10^{-3}$ (for a full compilation of all observational results see Rhee, et al. 2018). The HI density at $z=0$ is only $1.5 \times$ smaller than at $z=2$ with the data consistent with a no-evolution picture. On the other hand the cosmic SFRD has decreased 10 fold in this interval. Clearly our picture of galaxy formation is incomplete at these redshifts since the decrease in SFRD is not commensurate with a depletion of HI. Upcoming HI surveys like the Square Kilometre Array (SKA) will help us in this direction.

However even with the existing data it is important to understand the dependence of the abundance and distribution of HI selected galaxies on different galaxy properties and environments. The clustering of HI selected galaxies in the ALFALFA survey has been measured (Martin, et al. 2012; Papastergis, et al. 2013; Guo, et al. 2017) suggesting that ALFALFA galaxies cluster weakly. The dependence of HIMF has also been explored on the environment with Jones, et al. (2018) reporting a decrease in the low mass slope with increasing density in ALFALFA whereas Said, Kraan-Korteweg \& Staveley-Smith (2019) find that the low mass slope increases with increasing density in the HI Zone of Avoidance survey with the Parkes telescope. Similarly the the HI velocity width function has been studied for wall and void galaxies as well as red and blue galaxies (Moorman, et al. 2014), indicating a strong dependence on both environment and galaxy color. Zwaan, et al. (2003) have looked at the dependence of the HIMF on galaxy morphology as well as on the early-late type classification. In this paper we look at the dependence of the HIMF on the different

\footnotetext{
1 http://cesam.lam.fr/zCosmos/

2 https://cesam.lam.fr/vvds/

3 http://deep.ps.uci.edu/
} 
populations classified from the color-magnitude plane. This is similar in spirit to the analysis of Zwaan, et al. (2003); Moorman, et al. (2014) carried out in HI surveys and also bears resemblance to similar analysis carried out for optical surveys where the contribution of the red and blue galaxies to the galaxy stellar mass function and luminosity functions are explored (Baldry, et al. 2004; Drory, et al. 2009).

Our paper is organized as follows. In section 2 we describe a sample common to ALFALFA and SDSS (Sloan Digital Sky Survey), in section 3 we report our measurements of the HIMF for different populations within this sample, in section 4 we discuss our results and their implication and finally we summarize and conclude in section 5 . We assume a flat $\Lambda$ CDM cosmology with $\Omega_{m}=0.3, \Omega_{\Lambda}=0.7$ and a value for the dimensionless Hubble constant $h_{70}=0.7$.

\section{DATA}

At $40 \%$ data release (Haynes, et al. 2011) ALFALFA has surveyed over $\sim 2752 \mathrm{deg}^{2}$ of the sky, with comoving volume of $\sim 2.65 \times 10^{6} \mathrm{Mpc}^{3}$. Covering $40 \%$ of the targeted survey area, the $\alpha .40$ catalog contains 15855 sources in the regions $7^{h} 30^{m}<$ R.A. $<16^{h} 30^{m}, 4^{\circ}<$ dec. $<16^{\circ}$, and $24^{\circ}<$ dec. $<28^{\circ}$ and $22^{h}<$ R.A. $<3^{h}, 14^{\circ}<$ dec. $<16^{\circ}$, and $24^{\circ}<$ dec. $<32^{\circ}$. Most of these objects have optical counterparts in the Sloan Digital Sky Survey (SDSS) Data Release 7 (DR7) (Abazajian, et al. 2009).

This catalog contains the following observed quantities (i) an unique entry number from the Arecibo General Catalog(AGC); (ii) right ascension and declination of the HI source and the most probable optical counterpart (OC) from SDSS DR7; (iii) heliocentric velocity ( $\left.c z_{\text {helio }}\right)$ which is the midpoint of the HI flux density profile; (iv) velocity profile width $\left(W_{50}\right)$, measured as the full width at $50 \%$ of the peak HI flux density; (v) the integrated flux density of the HI source $\left(S_{21}\right)$. The derived quantities, contained in this catalog, are - (i) distance to the object in Mpc (D). For sources with $c z_{\text {helio }}>6000 \mathrm{~km} \mathrm{~s}^{-1}$ this quantity is $c z_{\mathrm{CMB}} / H_{0}$, where $c z_{\mathrm{CMB}}$ is the velocity in the cosmic microwave background (CMB) reference frame and $H_{0}$ is the Hubble constant (taken to be $70 \mathrm{~km} \mathrm{~s}^{-1} \mathrm{Mpc}^{-1}$ ). For sources with $c z_{\text {helio }}<6000 \mathrm{~km} \mathrm{~s}^{-1}$ this quantity has been estimated using a local flow model (Masters 2005) (ii) the HI mass $\left(M_{\mathrm{HI}}\right)$, computed as

$$
\frac{M_{\mathrm{HI}}}{M_{\odot}}=2.356 \times 10^{5}\left(\frac{D}{\mathrm{Mpc}}\right)^{2}\left(\frac{S_{21}}{\mathrm{Jy} \cdot \mathrm{km} \cdot \mathrm{s}^{-1}}\right)
$$

The $\alpha .40$ catalog also provides another property which is the Code number, with a value of 1,2 or 9 . The sources with SNR $>6.5$ are referred as Code 1 objects, Code 2 objects are the detections with $\mathrm{SNR}<6.5$ and Code 9 refers to the high velocity clouds (HVC).

For our analysis we have considered only Code 1 galaxies. The number of Code 1 galaxies is 11941 . We have also considered a cut for $c z_{\mathrm{CMB}}<15000 \mathrm{~km} . \mathrm{s}^{-1}$ to avoid radio frequency interference (RFI) generated by Federal Aviation Administration (FAA) radar at the San Juan airport (Martin, et al. 2010; Haynes, et al. 2011). This restricts the sample to redshift, $z \leq 0.05$ and reduces the sample to 10785 galaxies.

Since ALFALFA is not totally overlapping with the

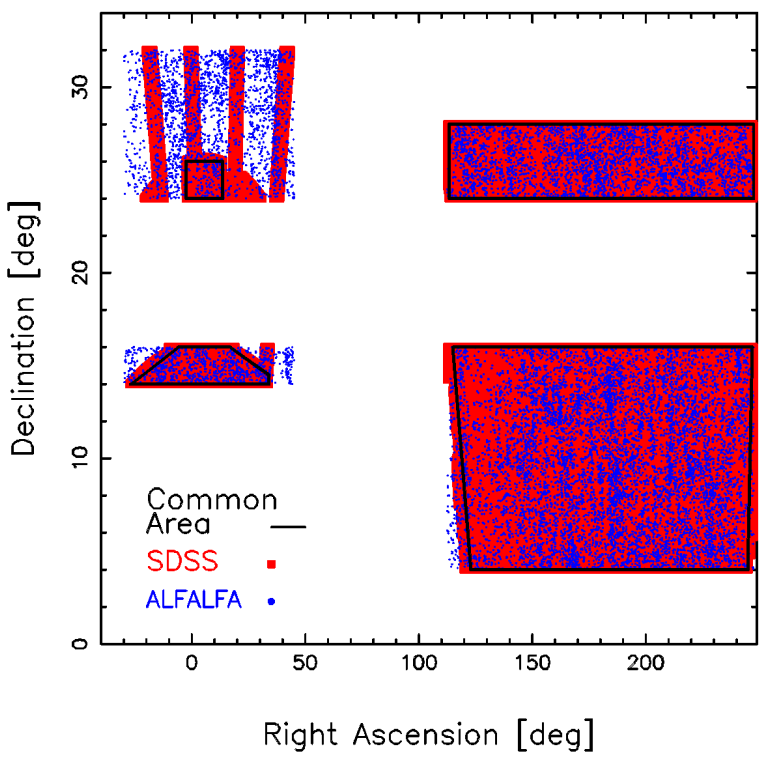

Figure 1. Common footprint of SDSS and ALFALFA used in this work. The pale grey patches is that of SDSS DR7 overlapping with the ALFALFA survey region. The black dots are the positions of ALFALFA galaxies. The common boundary used in this work is outlined by the thick black line.

SDSS footprint, we have defined a common boundary for both the surveys in this work in figure 1. For this work our final area of analysis has four subregions whose vertices right ascension-declination (RA,dec) are given as (i) $\left(123^{\circ}, 4^{\circ}\right)$, $\left(245^{\circ}, 4^{\circ}\right), \quad\left(247^{\circ}, 16^{\circ}\right), \quad\left(115^{\circ}, 16^{\circ}\right) ; \quad$ (ii) $\left(113.31^{\circ}, 24^{\circ}\right)$, $\left(247.5^{\circ}, 24^{\circ}\right),\left(247.5^{\circ}, 28^{\circ}\right),\left(113.31^{\circ}, 28^{\circ}\right)$; (iii) $\left(-27^{\circ}, 14^{\circ}\right)$, $\left(34^{\circ}, 14^{\circ}\right), \quad\left(34^{\circ}, 14.5^{\circ}\right), \quad\left(17^{\circ}, 16^{\circ}\right), \quad\left(-6^{\circ}, 16^{\circ}\right) ;$ and (iv) $\left(-2.6^{\circ}, 24^{\circ}\right),\left(13.6^{\circ}, 24^{\circ}\right),\left(13.6^{\circ}, 26^{\circ}\right),\left(-2.6^{\circ}, 26^{\circ}\right)$. The total volume of these regions is $2.02 \times 10^{6} \mathrm{Mpc}^{3}$ and it corresponds to an angular area of $\sim 2093 \mathrm{deg}^{2}$. In this region there are 8344 galaxies in ALFALFA. This includes removal of $4 \mathrm{OH}$ high-redshift impostors (Suess et al. 2016).

In figure 2 we look at the distribution of Code 1 objects in ALFALFA in the $S_{21}-W_{50}$ plane. We also show the distributions in three mass bins which correspond to the small-mass end or faint-end (thick solid line), the knee (dot-dashed line) and the high-mass end (thin-solid line). As seen from these distributions on average the velocity width increases with increasing mass. This is expected and is also seen in the $M_{\mathrm{HI}}-W_{50}$ relation (see figure 7 of Moorman, et al. 2014). One also sees that it is very unlikely to have a low(high) mass galaxy with a large(small) velocity width. However intermediate mass objects can have the full range of velocity widths.

The broken solid line in figure 2 is the sensitivity limit and is given by a $50 \%$ completeness relation of Code 1 objects in eq. 2 (Haynes, et al. 2011). This tells us that the detection of objects not only depends on the integrated flux, but also on the observed velocity width. At fixed $S_{21}$ the 


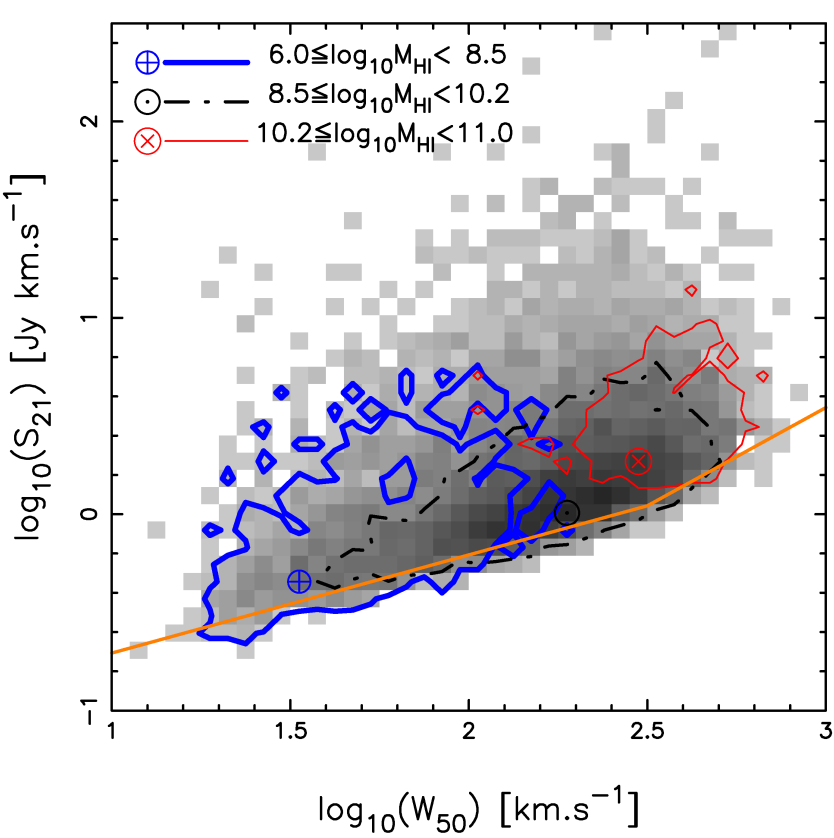

Figure 2. The distribution of galaxies in the $S_{21}-W_{50}$ plane is shown for all Code 1 ALFALFA galaxies. The broken solid line is the $50 \%$ completeness relation (Haynes, et al. 2011). It is given in eq. 2. We have further divided the sample into three mass bins: $\log _{10}\left[M_{\mathrm{HI}} / M_{\odot}\right] \in[6.0,8.5[,[8.5,10.2[,[10.2,11.0[$. The $1 \sigma$ contours and the peaks of the distributions for these three populations are given by thick solid line (plus-circle), the dotdashed line (dot-circle) and the thin solid line (cross-circle).

detection is more likely for narrower HI profile widths.

$\log S_{21}= \begin{cases}0.5 \log W_{50}-1.207 & : \log W_{50}<2.5 \\ \log W_{50}-2.457 & : \log W_{50} \geq 2.5\end{cases}$

After applying this completeness cut we are left with a sample of 7857 galaxies. Among these, 6076 galaxies have spectroscopic as well as photometric measurements in SDSS DR7 and 1633 galaxies have only photometric measurements. As for the remaining 148, we loosely refer to them as dark galaxies. These objects are not being identified in the SDSS pipeline as potential galaxies. Although follow up observations have been made on some of the dark galaxies (Cannon, et al. 2015; Janowieki, et al. 2015; Leisman, et al. 2017), in this work we refer to all of them as dark. This translates to around $\sim 2 \%$ of galaxies which are dark or have no optical counterparts in SDSS.

The ALFALFA catalog also lists the SDSS objectIDS of the OC. Using these we extracted the photometric properties like the ugriz values (model magnitudes), then corrected for extinction (due to our own galaxy) (Schlegel, Finkbeiner \& Davis 1998) for these 7709 (non-dark) galaxies. We also kcorrect (Blanton \& Roweis 2007) the magnitudes to finally obtain rest frame magnitudes. For objects which do not have spectroscopic redshifts we have supplied the HI redshifts to kcorrect them. The kcorrect code also estimates additional properties like galaxy stellar mass, integrated star formation history and metallicity for these objects.

In figure 3 we look at the observed distribution of galaxies both in SDSS and ALFALFA in the color $(u-r)$ magnitude $\left(M_{r}\right)$ plane. The $1 \sigma$ and $0.25 \sigma$ contours are given by the thin and thick lines. One can see a clear bimodality in the observed distribution in SDSS. The solid curve (eq. 3) is used to classify the galaxies into red (above curve) and blue (below curve) populations (Baldry, et al. 2004). The numbers indicate the observed counts for the red and blue populations in this color-magnitude range. As discussed earlier $\sim 98 \%$ of ALFALFA galaxies have optical counterparts in SDSS. Here we show that ALFALFA predominantly samples the blue cloud. We see that about $38 \%(11 \%)$ of blue(red) galaxies in SDSS have detections in ALFALFA.

As of writing this paper the ALFALFA team has released the $100 \%$ catalog $(\alpha .100)$ (Haynes, et al. 2018) which also include the RA and dec of the optical counterparts. However we find that there are many galaxies which have luminous foreground stars due to which SDSS has masked the region covering the galaxy and photometric values are not provided. We therefore restrict ourselves with $\alpha .40$ catalog and will revisit the $\alpha .100$ sample in the future.

\subsection{Subsamples and Populations of Galaxies}

From our sample of 7857 galaxies we identify subsamples which define different populations of galaxies. These populations are disjoint sets and their union (including the dark galaxies) forms the full sample. The populations are based on dividing the color $(u-r)$-magnitude $\left(M_{r}\right)$ plane of the HI selected galaxies into six disjoint regions. As seen in figure 3 there are two distinct populations, red and blue, seen in SDSS. We will start with this definition to further break our full sample into different populations. We show this in figure 4 (which is similar to figure 3 ), where we plot individual points instead of binning the data.

In figure 4 the upper solid curve demarcates the red (above curve) from the blue (below curve) population as in Baldry, et al. (2004). This optimal divider is given by

$C_{u r}^{\prime}\left(M_{r}\right)=2.06-0.244 \tanh \left[\frac{M_{r}+20.07}{1.09}\right]$

The vertical solid line divides the luminous (leftward of line) and intrinsically faint populations (henceforth we will call these faint populations). The line has been chosen so that the fraction of luminous red objects over all red objects is 0.87 which we refer to as the $1.5 \sigma$ line. Similarly the lower solid curve is chosen so that the fraction of blue (above curve) galaxies over all blue galaxies is 0.87 (or the $1.5 \sigma$ cut in color). This curve has been chosen to be parallel to the curve which demarcates the red and blue populations. We refer to the objects below(above) the curve as bluer(blue) galaxies. Similarly we define the $1 \sigma$ sample (dashed lines). This breaks the sample of HI selected galaxies (which have optical counterparts) into 6 disjoint sets in the color-magnitude plane; we call each set a single population. The number for each population is quoted. The numbers in brackets are for the $1 \sigma$ sample. The data points are: i. filled (open) triangles for luminous (faint) red galaxies ii. filled (open) squares for luminous (faint) blue galaxies iii. filled (open) circles for luminous (faint) bluer galaxies and have been marked for the $1.5 \sigma$ sample.

Our definition of a population of galaxies is broadly a subsample of the total sample of $7857 \mathrm{HI}$ selected galaxies. The dark population has no optical counterparts. The luminous(faint) population is a subsample of galaxies with ab- 

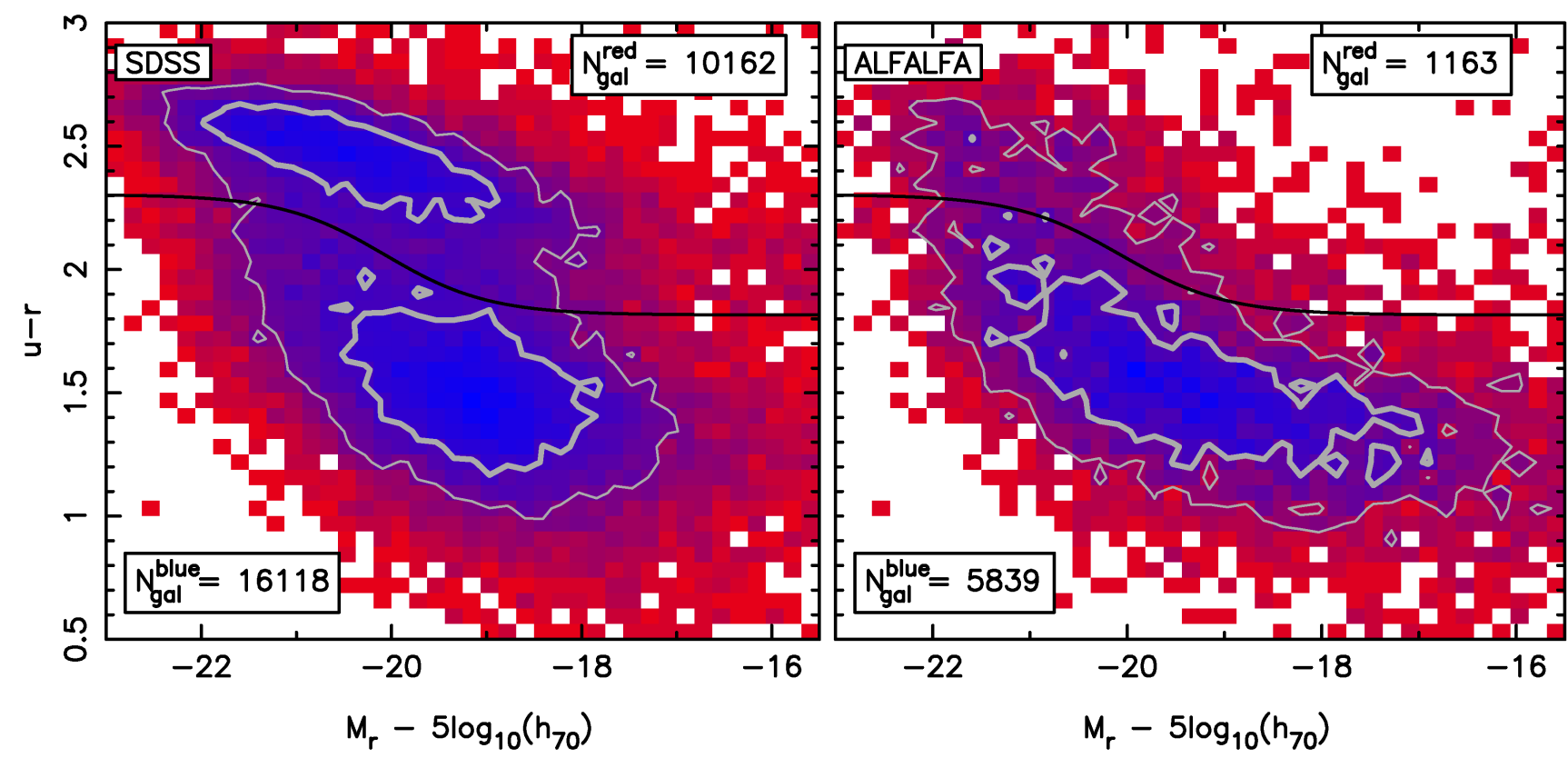

Figure 3. The observed distribution of galaxies in the color-magnitude plane for SDSS (left panel) and ALFALFA (right panel) in a common volume considered in this work. The $1 \sigma$ and $0.25 \sigma$ contours are given by the thin and thick lines. A double peaked, bimodal distribution of galaxies is visible in SDSS and the solid curve (eq. 3) is used to classify the galaxies into red (above curve) and blue (below curve) populations (Baldry, et al. 2004). The numbers indicate the observed counts of galaxies in this color-magnitude range for each of the populations.

solute magnitudes $M_{r}^{i}$, such that $M_{r}^{i}<M_{r}\left(M_{r}^{i} \geq M_{r}\right)$ for some reference value $M_{r}$ (the vertical line in figure 4 ). Similarly we have defined three populations based on color (red, blue and bluer), based on equation 3. The six populations are formed by further splitting these three into luminous and faint populations. As discussed earlier, each population is dependent on the boundaries that define them which we have called a $1 \sigma$ or $1.5 \sigma$ line. We caution the reader that our definition of sample may be confusing since both these samples represent the same set of 7709 galaxies which have optical counterparts. However the number of galaxies for any given population is different for the $1 \sigma$ and $1.5 \sigma$ samples. For the $1.5 \sigma$ sample the fraction of detections of ALFALFA with respect to SDSS for the luminous red, blue and bluer populations are $11 \%, 32 \%, 39 \%$ and it is $14 \%, 58 \%, 62 \%$ for the faint populations.

We have chosen to define two samples to demonstrate that the qualitative results do not depend very strongly on sample definition. However as we will show in the next section, the definition of the faint bluer population (i.e. the $1.5 \sigma$ or $1 \sigma$ sample) determines whether it contributes significantly or not to some part of the HIMF. Looking at how the observed counts change, both in relative and absolute terms, when going from the $1.5 \sigma$ to the $1 \sigma$ sample we expect that the HIMF of the luminous blue population to be the affected the most. In section 4 (figure 7) we will discuss the break in the $M_{\mathrm{HI}}-M_{\text {star }}$, at $M_{\text {star }} \simeq 9$, where $M_{\text {star }}$ is the logarithm of the galaxy stellar mass in units of $M_{\odot} / h_{70}^{2}$. One can then use the mean $M_{r}-M_{\text {star }}$ relation to convert $M_{\text {star }}=9$ to $M_{r}=-19$. This value is sandwiched between the vertical lines (solid and dashed) in figure 4.

\section{RESULTS}

The mass function $\phi(M)$ is defined as the number density of objects in the mass range $[M, M+d M]$. The HI mass function $\phi\left(M_{\mathrm{HI}}\right)$ can be expressed as

$$
\phi\left(M_{\mathrm{HI}}\right)=\frac{d N}{V d M_{\mathrm{HI}}}
$$

where, $d N$ is the total number of galaxies having HI mass between $M_{\mathrm{HI}}$ and $M_{\mathrm{HI}}+d M_{\mathrm{HI}}$ and $V$ is the survey volume of interest. The HI mass function can be parameterized as a Schechter Function

$$
\phi\left(M_{\mathrm{HI}}\right)=\phi_{*}\left(\frac{M_{\mathrm{HI}}}{M_{*}}\right)^{\alpha} \exp \left(-\frac{M_{\mathrm{HI}}}{M_{*}}\right)
$$

Here, $\alpha$ is the faint end slope, $\phi_{*}$ is the amplitude and $M^{*}$ is the characteristic HI mass.

A simple and intuitive way of calculating the HIMF is by the $1 / V_{\max }$ method (Schmidt 1968). The underlying assumption in this method is that the sample of galaxies detected by a survey is a representative sample of galaxies in the Universe (in the same redshift range), or in other words it assumes homogeneity. For each detected galaxy ' $i$ ', a maximum detectable distance $D_{\max }^{i}$ is calculated based on inverting eq. 1 by using the mass $M_{\mathrm{HI}}^{i}$ of the galaxy and the limiting flux $S_{\lim }^{i}$ which is the property of the survey. In the case of ALFALFA the completeness relation (eq. 2) determines this limiting flux for the velocity width $W_{50}^{i}$ of the galaxy. $D_{\max }^{i}$ is then converted to a volume $V_{\max }^{i}$ which is the volume in which the galaxy could be in, and still be detected by the survey. Finally the galaxies are binned in mass with relative weights $1 / V_{\max }^{i}$ to obtain the mass function. For the more luminous or more massive objects $V_{\max }^{i}$ is larger than the survey volume. In such a case the relative 


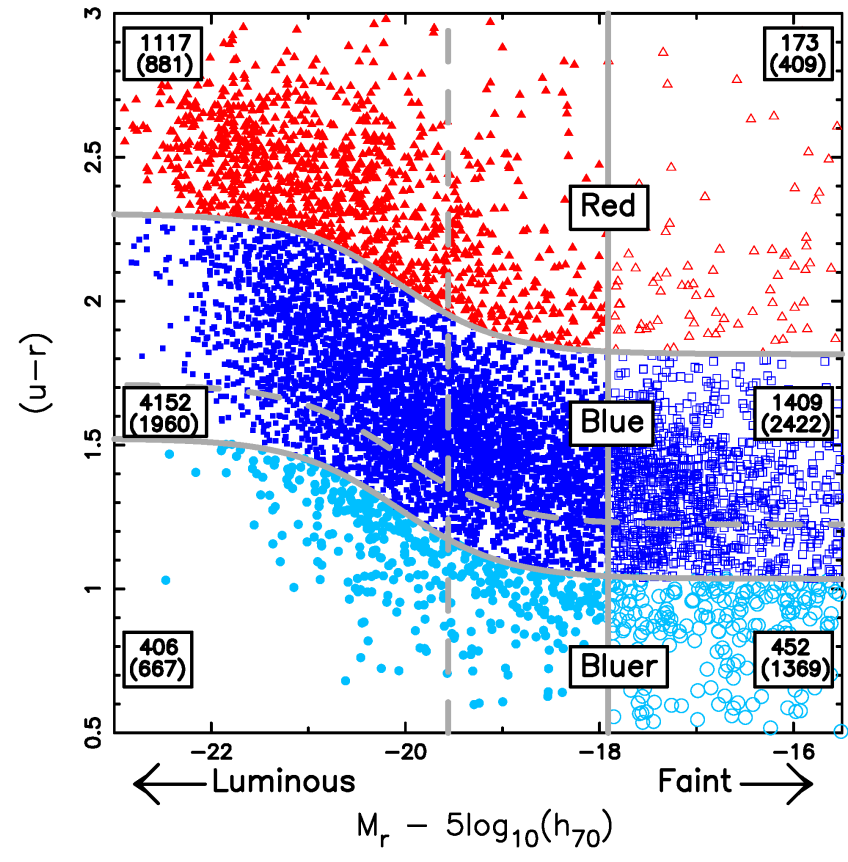

Figure 4. The distribution of HI selected galaxies in this work in the color-magnitude plane. The upper solid curve demarcates the red (above curve) from the blue (below curve) population as in Baldry, et al. (2004). The vertical solid (dashed) line divides the luminous and faint populations. The line has been chosen so that the fraction of luminous red objects over all red objects is 0.87 which we refer as the $1.5 \sigma$ line. Similarly the lower solid curve is chosen so that the fraction of blue (above curve) galaxies over all blue galaxies is 0.87 (or the $1.5 \sigma$ cut in color). This curve has been chosen to be parallel to the curve which demarcates the red and blue populations. We refer to the objects below(above) the curve as bluer(blue) galaxies. Similarly we define the $1 \sigma$ sample with the help of dashed lines. This breaks the sample of HI selected galaxies (which have optical counterparts) into 6 disjoint sets in the color-magnitude plane. The number for each population is quoted. The numbers in brackets are for the $1 \sigma$ sample.

weight is set to unity. This method has the advantage that it is non-parametric and does not require any prior knowledge to estimate the HIMF. However since galaxies cluster, the estimate of the HIMF will be sensitive to large scale structure in the local volume. Additionally some volumes of the survey may be inaccessible due to RFI and needs further correction. An estimate of these effects can be used to recalibrate the weights $V_{\max }^{i}$ (Martin, et al. 2010).

Maximum likelihood (Sandage, Tammann, \& Yahil 1979) and Step-Wise Maximum Likelihood (SWML) (Efstathiou, Ellis, \& Peterson 1988) methods on the other hand are designed to be insensitive to large-scale structure. In the former the assumption is that the galaxy sample is drawn from an underlying distribution function, e.g. the HIMF for this work $\phi\left(M_{\mathrm{HI}}\right)$, and the likelihood method determines the parameters of this function. Although in most cases a Schechter function is the chosen function one has no way of testing whether it is the optimal function to describe data (Efstathiou, Ellis, \& Peterson 1988). Baldry, et al. (2012) and Drory, et al. (2009) find that a single Schechter function does not describe the galaxy stellar mass function. In the latter, i.e. SWML, the underlying distribution $\phi\left(M_{\mathrm{HI}}\right)$ has no functional form but is discretized in steps or bins of mass and a uniform distribution is assumed in each bin. Hence the value $\phi_{j}$ which is the value of $\phi$ in the $j^{\text {th }}$ mass bin becomes the parameter. The joint likelihood of detecting all galaxies in the sample is maximized, with respect to the parameters $\phi_{j}$, thus determining their values. This method works when the sample is flux-limited.

When the selection function depends on other properties of the galaxies one needs to consider an underlying bivariate or multivariate distribution for $\phi$. One has to then generalize the SWML method to higher dimensions. Loveday (2000) estimated the bivariate luminosity function $\phi\left(M_{\mathrm{K}}, M_{\mathrm{B}}\right)$ and then marginalized over $M_{\mathrm{B}}$ to obtain the K-Band luminosity function, $\phi\left(M_{\mathrm{K}}\right)$, starting with a $b_{J^{-}}$ selected sample in the Stromlo-APM Redshift Survey.

For a blind HI survey like ALFALFA the limiting flux (figure 2 and eq. 2) depends on the velocity width $W_{50}$. A two-dimensional SWML (2DSWML) method similar to Loveday (2000) was applied by Zwaan, et al. (2003) to estimate the HIMF for HIPASS Galaxies. The bivariate distribution in this case is $\phi\left(M_{\mathrm{HI}}, W_{50}\right)$ which can be marginalized over $W_{50}$ to obtain the HIMF (Zwaan, et al. 2003; Martin, et al. 2010; Haynes, et al. 2011; Jones, et al. 2018) or marginalized over $M_{\mathrm{HI}}$ to obtain the HI velocity width function (Zwaan, Meyer, \& Staveley-Smith 2010; Moorman, et al. 2014). The details of our implementation are given in appendix A.

In figure 5 we show our estimate of the HIMF for all the populations including the total (crosses) and the dark (filled diamonds) populations. The columns are for the $1.5 \sigma$ (left) and the $1 \sigma$ (right) populations. We have broken our results into a dominant (top row) and a subdominant (bottom row) population to better illustrate our results. We call a dominant population one which dominates the HIMF over the rest of the populations in some mass range and also contributes greater than $10 \%$ to $\Omega_{\mathrm{HI}}^{\text {tot }}$ (see table 2 ). The symbols for the six populations are the same as in figure 4 . The curves are Schechter function fits for the total (solid), red (dotted), blue (dot-dashed), bluer (dashed) and dark (dot-dot-dotdashed) populations. Our Schechter function fits are summarized in table 1 . In the rest of the paper we will quote the values of the characteristic mass $M_{*}$ and the amplitude of the Schechter function $\phi_{*}$ in the units $\log \left(M_{*} / M_{\odot}\right)+2 \log h_{70}$ and $\left(10^{-3} h_{70}^{3} M p c^{-3} \mathrm{dex}^{-1}\right)$ respectively. We will also quote the values of $M_{\mathrm{HI}}$ in the same units as $M_{*}$. In practice we bin the mass and the velocity width in logarithmic bins therefore the faint slope in figure $5\left(\alpha^{\prime}\right)$ differs from $\alpha$ in equation 5 by 1 , or $\alpha^{\prime}=\alpha+1$.

Since we are working with Code 1 objects in the $\alpha .40$ sample it is appropriate to compare the total HIMF to that of Martin, et al. (2010). For the HIMF we find that our results of $\left(M_{*} \pm \sigma_{M_{*}}, \phi_{*} \pm \sigma_{\phi_{*}}, \alpha \pm \sigma_{\alpha}\right)=(9.96 \pm 0.02,5.34 \pm$ $0.40,-1.35 \pm 0.02)$ and $\chi_{\text {reduced }}^{2}=0.79$ (table 1$)$. This is consistent at the $1 \sigma$ level with Martin, et al. (2010) who found $\left(M_{*} \pm \sigma_{M_{*}}, \phi_{*} \pm \sigma_{\phi_{*}}, \alpha \pm \sigma_{\alpha}\right)=(9.96 \pm 0.02,4.8 \pm$ $0.30,-1.33 \pm 0.02)$. Note however that $\phi_{*}$ is barely within $1 \sigma$ of each other. We attribute this difference to the choice of sample in this work which has $\sim 25 \%$ fewer galaxies than Martin, et al. (2010). We point out that when we consider the full sample our results match well (see e.g. figure A3).

The goodness of fits, $\chi_{\text {reduced }}^{2}$, is given in the last column of table 1. For the dark, faint red, faint bluer populations the $\chi_{\text {reduced }}^{2}$ is of order unity. In the $1 \sigma$ sample of the faint 

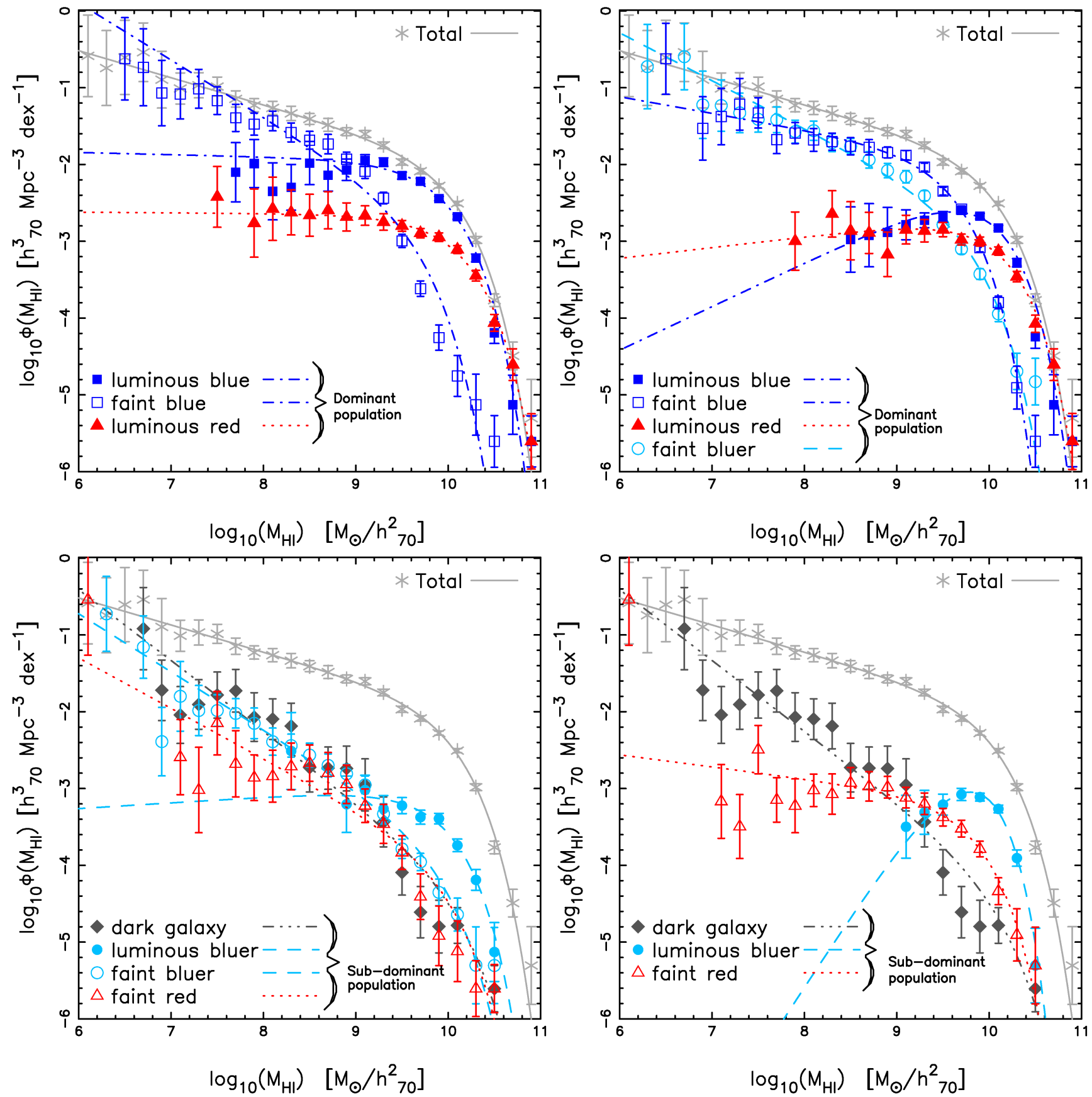

Figure 5. The HIMF for the $1.5 \sigma$ (left column) and the $1 \sigma$ (right column) samples. Data points and error bars were estimated using the 2DSWML method (see appendix A). The curves are Schechter function fits. For comparison we have added the total HIMF (crosses) and its fit (solid line) in all the panels. For each sample the top row is for the dominant population and the bottom for the sub-dominant one. In addition to the six populations we have also added the HIMF for the dark population (filled diamonds) and its Schechter function fit (dot-dot-dot-dashed line). The symbols for the six populations are the same as in figure 4 . The Schechter fits are given for the red (dotted), blue (dot-dashed) and bluer (dashed) populations. The details of the fits are given in table 1 .

red population the biggest contribution of $\chi_{\text {reduced }}^{2}$ comes from the lowest mass bin. If we remove that point as an outlier then $\chi_{\text {reduced }}^{2}=0.57$ and $\alpha$ flattens to -1.06 from $-1.16, \phi_{*}$ increases by about $22 \%$ from 0.31 . The change in $M_{*}$ is negligible. The luminous red and luminous bluer populations have a low $\chi_{\text {reduced }}^{2}$, irrespective of sample definition, and looking at the data points relative to their fitted curve we find that there is little variation between them.
This means that the error bars are larger than the variation between data and model. The luminous blue and faint blue populations on the other hand have larger $\chi_{r \text { reduced }}^{2}$. For the $1.5 \sigma$ sample of the faint blue population $\chi_{\text {reduced }}^{2}=4.10$ and improves to $\chi_{\text {reduced }}^{2}=0.96$ for the $1 \sigma$ sample. The $1 \sigma$ sample for the faint blue population has a larger number of luminous galaxies as compared to the $1.5 \sigma$ sample (figure 4 ) and because $M_{\mathrm{HI}}$ is correlated to $M_{r}$, (see figure 6) the high 


\begin{tabular}{lcccc}
\hline region & $\log \left(M_{*} / M_{\odot}\right)+2 \log h_{70}$ & $\left(10^{-3} h_{70}^{3} M p c^{-3} \mathrm{dex}^{-1}\right)$ & $\alpha$ & $\chi_{\text {reduced }}^{2}$ \\
\hline total & $9.96 \pm 0.02$ & $5.34 \pm 0.40$ & $-1.35 \pm 0.02$ & 0.79 \\
\hline luminous blue & $9.86 \pm 0.02(9.85 \pm 0.03)$ & $4.85 \pm 0.42(2.54 \pm 0.17)$ & $-1.03 \pm 0.06(-0.43 \pm 0.11)$ & $2.02(2.17)$ \\
\hline faint blue & $9.57 \pm 0.04(9.50 \pm 0.02)$ & $1.22 \pm 0.22(5.84 \pm 0.58)$ & $-1.74 \pm 0.05(-1.22 \pm 0.05)$ & $4.10(0.96)$ \\
\hline luminous red & $10.04 \pm 0.04(10.02 \pm 0.04)$ & $0.96 \pm 0.12(0.95 \pm 0.12)$ & $-1.01 \pm 0.08(-0.86 \pm 0.10)$ & $0.32(0.52)$ \\
\hline luminous bluer & $9.84 \pm 0.08(9.52 \pm 0.05)$ & $0.46 \pm 0.13(0.79 \pm 0.16)$ & $-0.92 \pm 0.23(0.86 \pm 0.38)$ & $0.64(0.38)$ \\
\hline faint bluer & $9.83 \pm 0.07(9.72 \pm 0.04)$ & $0.10 \pm 0.03(1.09 \pm 0.18)$ & $-1.76 \pm 0.06(-1.62 \pm 0.05)$ & $1.11(1.40)$ \\
\hline faint red & $9.89 \pm 0.07(9.74 \pm 0.06)$ & {$[6.09 \pm 2.07] \times 10^{-2}(0.31 \pm 0.07)$} & $-1.66 \pm 0.11(-1.16 \pm 0.08)$ & $1.27(1.36)$ \\
\hline dark & $10.03 \pm 0.09$ & {$[3.25 \pm 1.50] \times 10^{-2}$} & $-1.92 \pm 0.09$ &
\end{tabular}

Table 1. Parameters of the Schechter function fit to the HIMF for all the populations. The estimated parameters and their uncertainties are for the $1.5 \sigma$ sample and the numbers in brackets are for the $1 \sigma$ sample. The goodness of fit, $\chi_{\text {reduced }}^{2}$ is given in the last column.

mass end of the HIMF is better represented in the $1 \sigma$ sample, leading to a smoother change in data and a better fit. This can also be seen in the top row of figure 5 . The luminous blue population has $\chi_{\text {reduced }}^{2} \sim 2$ irrespective of sample definition.

We start by looking at the luminous population. Due to the monotonic relation between $M_{\mathrm{HI}}$ and $M_{r}$ (figure 6 ), across populations, we expect the luminous population to dominate the massive end of the HIMF and be subdominant at the low mass end. This is seen in figure 5. The characteristic mass, $M_{*}$ increases systematically from the luminous bluer to the luminous red population. There is little change in $M_{*}$ for both the luminous red and luminous blue populations with respect to sample definition. We also see little change in the HIMF with respect to sample definition for $M_{\mathrm{HI}} \geq 10.3$ for both these populations. The luminous bluer population on the other hand has $M_{*}=9.84$ for the $1.5 \sigma$ sample and reduces to $M_{*}=9.52$ for the $1 \sigma$ sample. At the low mass end the luminous populations have shallower slopes $\alpha+1 \geq 0$. This is expected as mentioned earlier since at the low mass end we expect the faint population to dominate. At this end, the sample definition affects the luminous bluer population the most, where $\alpha=-0.92$ ( $1.5 \sigma$ sample) and increases to $\alpha=+0.86$ ( $1 \sigma$ sample), the change being the smallest for the luminous red population. On the other hand the amplitude is most affected for the luminous blue population, it changes from $\phi_{*}=4.85$ ( $1.5 \sigma$ sample) to $\phi_{*}=2.54(1 \sigma$ sample). The change is negligible for the luminous red and about $\sim 72 \%$ for the luminous bluer population. We point out that changes in the HIMF with respect to sample definition can be best understood in terms of how the observed number of galaxy populations change when the boundaries in the color-magnitude plane are redrawn to define a new sample (figures 4) and the average scaling relation $M_{\mathrm{r}}-M_{\mathrm{HI}}$ (figure 6$)$. For the $1.5 \sigma$ sample the luminous blue is the dominant population at the knee of the HIMF, however it contributes nearly equally as the faint blue population when we consider the $1 \sigma$ sample. This is because the faint blue population has a net increase in observed galaxies from $n_{\text {gal }}=1409$ ( $1.5 \sigma$ sample $)$ to $n_{\text {gal }}=2422(1 \sigma$ sample $)$, the net change coming from the intersection of luminous blue $(1.5 \sigma)$ and faint blue $(1 \sigma)$ (see figure 4 ); this is the primary reason for the increase of $\phi_{*}$ by $\sim 4.8 \times$. An interesting result is that the luminous red population is the dominant population at $M_{\mathrm{HI}} \geq 10.4$. This result is insensitive to sample definition. We find that for $M_{\mathrm{HI}} \geq 10.4$ the luminous red population represents $\sim 60 \%$ of total detections with $\sim 40 \%$ coming from the luminous blue population which also translates to similar fractions in total HI mass at this end.

We now move to the faint population. All of them have steeper slopes as compared to their luminous counterparts and do not dominate the HIMF at the high mass end. The faint red population is the most subdominant population. The observed counts of galaxies of the faint bluer population see the largest relative change with sample definition increasing from $n_{\text {gal }}=452(1.5 \sigma$ sample $)$ to $n_{\text {gal }}=1369(1 \sigma$ sample), a factor $\sim 3 \times$. This results in a small change in slope from $\alpha=-1.76$ to $\alpha=-1.62$ but a large, $\sim 10 \times$, increase in amplitude from $\phi_{*}=0.10$ to $\phi_{*}=1.09$. In the $1.5 \sigma$ sample the faint bluer population is a subdominant population, however it becomes the dominant population for masses $M_{\mathrm{HI}} \leq 8.1$. The faint blue population is on the other hand a dominant population below the knee of the mass function. It dominates the HIMF for $M_{\mathrm{HI}} \leq 8.7$ in the $1.5 \sigma$ sample and for $8.1 \leq M_{\mathrm{HI}} \leq 9.7$ in the $1 \sigma$ sample.

The dark population is characterized by a very steep slope $\alpha=-1.92$, large characteristic mass $M_{*}=10.03$ and a very small amplitude $\phi_{*}=3.25 \times 10^{-2}$ and is a subdominant population. Extrapolating to masses below $M_{\mathrm{HI}} \leq 6$ our results suggest the dark population will be the dominant population. However it is unclear how far down we can extrapolate since it is unlikely that there will be too many low mass, gas rich galaxies devoid of stars which will be able to self shield themselves from the photoionizing background.

\subsection{The contribution of different galaxy populations to $\Omega_{\mathrm{HI}}$}

We can analytically integrate the HIMF, fitted to a Schechter function to obtain the cosmic HI density parameter,

$$
\Omega_{\mathrm{HI}}=\frac{\rho_{\mathrm{HI}}}{\rho_{c}}=\frac{M_{*} \phi_{*}}{\rho_{c}} \Gamma(\alpha+2)
$$




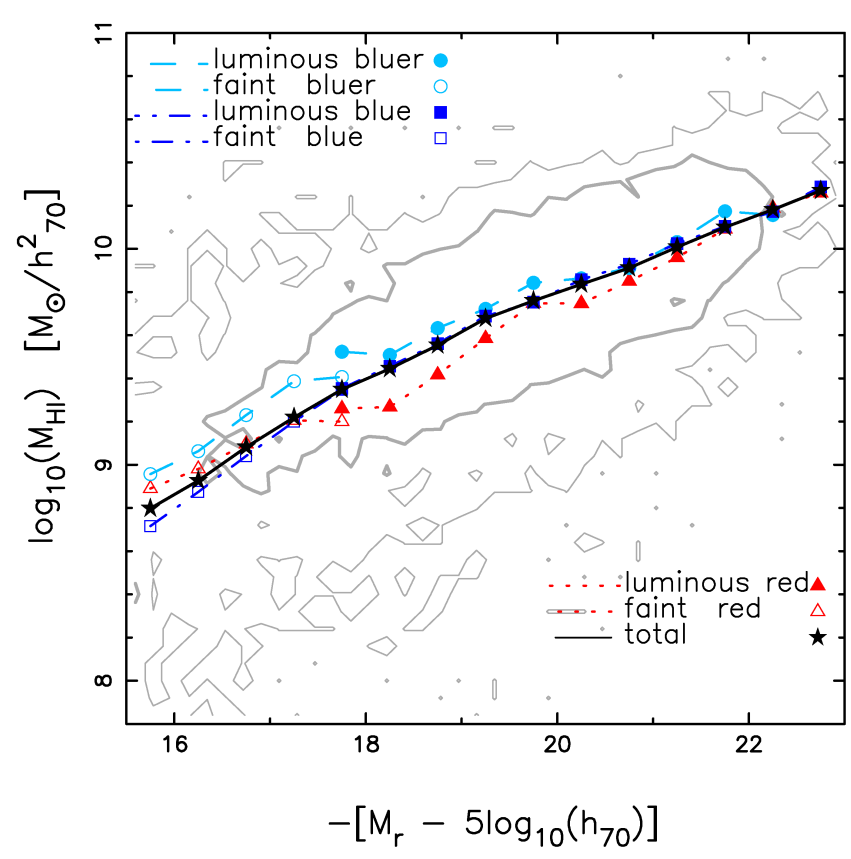

Figure 6. The $M_{\mathrm{r}}-M_{\mathrm{HI}}$ relation for the $1.5 \sigma$ sample. The filled star represents the total sample excluding the dark galaxies. The other data points and line styles are the same as in figure 5.

Alternately we can sum the binned measurements of the HIMF. Similar to Haynes, et al. (2011) we find that both methods give similar results, with the exception of the dark sample which has a very steep slope. As we argued in the previous section it is not physical to extrapolate the HIMF to very small masses. Hence we choose to quote our results by the summed method. We note that the results do not change if we integrate the Schechter function from $M_{\mathrm{HI}}=$ 6.1 to $\infty$. We summarize our results in table 2 . Column 2 is the estimate of $\Omega_{\mathrm{HI}}$ from each population and column 3 is the fractional contribution to $\Omega_{\mathrm{HI}}^{\mathrm{tot}}$, the values in brackets are for the $1 \sigma$ sample.

For the total sample we obtain $\Omega_{\mathrm{HI}}=(4.894 \pm 0.469) \times$ $10^{-4} h_{70}^{-1}$ which is consistent at $1 \sigma$ with $\Omega_{\mathrm{HI}}=(4.4 \pm 0.1) \times$ $10^{-4} h_{70}^{-1}$ (summed) of Martin, et al. (2010). With the addition of Code 2 objects in the $\alpha .40$ sample we see that it is only consistent at the $2 \sigma$ level with Haynes, et al. (2011) who obtain $\Omega_{\mathrm{HI}}=(4.2 \pm 0.1) \times 10^{-4} h_{70}^{-1}$ (summed). Since our $M_{*}$ is comparable with the $\alpha .40$ results, and $\alpha$ is only a bit steeper, the main reason for this discrepancy can be traced to $\phi_{*}$ (equation 6). Our value of $\phi_{*}$ is $\sim 10 \%$ higher than Martin, et al. (2010) which translates to a $10 \%$ higher estimate of $\Omega_{\mathrm{HI}}$ at fixed $M_{*}$ and $\alpha$. However the relative ratios should not be sensitive to this change.

From table 2 we see that the red population (luminous and faint) have a non-negligible contribution of $\sim 17 \%$ to $\Omega_{\mathrm{HI}}^{\mathrm{tot}}$. When combined with the dark population $\left(3 \%\right.$ of $\left.\Omega_{\mathrm{HI}}^{\mathrm{tot}}\right)$, this adds up to a non-negligible fraction of $20 \%$ (rounded). The full blue population (faint and luminous blue and bluer) then contributes $\sim 80 \%$ (rounded) of $\Omega_{\mathrm{HI}}^{\text {tot }}$. We will discuss the implications of these numbers in the next section. The dominant sample about the knee of the HIMF are the luminous blue and the faint blue populations (as $M_{*}$ galaxies) and together they contribute most to $\Omega_{\mathrm{HI}}^{\text {tot }}, \sim 73 \%(1.5 \sigma$ sample) and $\sim 55 \%(1 \sigma$ sample $)$
In section 3 we defined a dominant population as one which dominates the total HIMF in some mass range and contributes more than $10 \%$ to $\Omega_{\mathrm{HI}}^{\text {tot }}$. For the $1.5 \sigma$ sample the dominant populations are the luminous red, luminous blue and faint blue populations. Whereas for the $1 \sigma$ sample the dominant populations are the luminous red, luminous blue, faint blue and faint bluer populations. The dominant populations together contribute about $90 \%$ to $\Omega_{\mathrm{HI}}^{\text {tot }}$, irrespective of sample definition. By integrating the HIMF for the dominant populations we find that they represent about $85 \%$ $(90 \%)$ of galaxies above $M_{\mathrm{HI}} \geq 8\left(M_{\mathrm{HI}} \geq 9\right)$ for the $1.5 \sigma$ sample. The numbers are similar for the $1 \sigma$ sample. We do not present numbers for a lower mass threshold (which would dominate the total number density) since all populations do not have detections at lower masses and the errorbars for the HIMF are considerably large at lower masses.

\section{DISCUSSION}

Since we are looking at the contribution of different galaxy populations to the total HIMF we would like to see whether these populations have different scaling relations, e.g. in the $M_{\mathrm{HI}}-M_{\text {star }}$ plane. Such relations have been explored for galaxies in the ALFALFA sample (Catinella, et al. 2010; Huang, et al. 2012; Maddox, et al. 2015) and the HI Parkes All-Sky Survey Catalog (HICAT) (Parkash, et al. 2018). In this work the stellar masses are estimated by kcorrect which uses the population synthesis code of Bruzual \& Charlot (2003). Our estimates on $M_{\text {star }}$ differ from the estimates of these authors. The $M_{\mathrm{HI}}-M_{\text {star }}$ scaling relations are shown in figure 7 .

In order to avoid crowding figure 7 we choose to compare our results for the total sample with Huang, et al. (2012) (crossed-circle, thin solid line) only. The $1 \sigma$ scatter on the data points (total) is $\sim 0.5$ dex. For the total sample we find that our results compare well (within the scatter) with Huang, et al. (2012) in figure 7. The scaling relations that we find are also consistent with Maddox, et al. (2015); Parkash, et al. (2018) ( $M_{\mathrm{HI}}$ selected sample) but differ from Catinella, et al. (2010) ( $M_{\text {star }}$ selected sample). However our stellar masses are underestimated at lower masses. This difference can be attributed to the choice of sample but more so due to attenuation by dust, affecting the redder sample, which these authors have considered. In this work we have not attempted to correct for reddening due to dust while Huang, et al. (2012) have used the additional two UV bands in GALEX to correct for it. Not correcting for it should therefore change the average scaling relations. This is also evident when looking at the scaling relations for the three faint populations. The faint blue and faint bluer populations have similar slopes but these are steeper compared to their corresponding luminous populations. The faint red population, on the other hand, has a shallower slope with respect to the luminous red population as well as the faint blue and bluer populations. We also find that the HI fraction, $f_{\mathrm{HI}}=M_{\mathrm{HI}} / M_{\mathrm{star}}$, increases with decreasing $(u-r)$ color.

We see a clear transition in the scaling relations when going from the low mass to the high mass end. $f_{\mathrm{HI}}$ gets suppressed for the total sample at about $M_{\text {star }} \sim 9$ consistent with Huang, et al. (2012); Maddox, et al. (2015). The transition scale also depends on the galaxy popula- 


\begin{tabular}{lcc}
\hline region & $\Omega_{H I}\left[10^{-4} h_{70}^{-1}\right]$ & $\Omega_{H I} / \Omega_{H I}^{\text {total }}$ \\
\hline total & $4.894 \pm 0.469$ & 1.00 \\
\hline luminous blue & $2.543 \pm 0.298(1.099 \pm 0.115)$ & $0.520(0.224)$ \\
\hline faint blue & $1.014 \pm 0.455(1.604 \pm 0.196)$ & $0.207(0.328)$ \\
\hline luminous red & $0.764 \pm 0.124(0.653 \pm 0.110)$ & $0.156(0.133)$ \\
\hline luminous bluer & $0.215 \pm 0.135(0.333 \pm 0.165)$ & $0.044(0.068)$ \\
\hline faint bluer & $0.167 \pm 0.126(0.957 \pm 0.233)$ & $0.034(0.196)$ \\
\hline faint red & $0.094 \pm 0.050(0.144 \pm 0.038)$ & $0.019(0.029)$ \\
\hline dark & $0.162 \pm 0.137$ & 0.033 \\
\hline
\end{tabular}

Table 2. The contribution of different populations to $\Omega_{\mathrm{HI}}$. Column 2 is the estimate of $\Omega_{\mathrm{HI}}$ from a single population and column 3 is the fractional contribution to $\Omega_{\mathrm{HI}}^{\mathrm{tot}}$. The estimated values for the $1 \sigma$ sample is in brackets.

tion. For the blue, bluer and red populations it occurs at $M_{\text {star }}=10.1,9.4,8$ respectively. The transition scale of $M_{\text {star }} \sim 9$ corresponds to a change in the dominant morphology of galaxy populations (Maddox, et al. 2015) and also a transition between hot and cold mode accretion seen in cosmological hydrodynamical simulations (Kereš, et al. 2009).

One interesting result that we have quantified in the last section is the non-negligible $\mathrm{HI}$ content of red galaxies. The red galaxies dominate the HIMF at the high mass end $M_{\mathrm{HI}} \geq 10.4$ and $\sim 17 \%$ of the HI content, $\Omega_{\mathrm{HI}}$, is locked up in them. Using the HOD framework for HI, Paul, Choudhury \& Paranjape (2018) also find that the red population is the dominant population at higher masses. Since the ALFALFA sample is an HI selected sample, with the majority of the galaxies belonging to the blue cloud, one may ask: why do the rarest, gas rich galaxies, predominantly belong to the red cloud? Looking at the morphology of these gas rich red galaxies we find that these are predominantly spirals and lenticular galaxies, but there also exist some elliptical galaxies. A number of spirals have prominent bulges which would classify them as early-type spirals and there are a number of galaxies which harbor dust lanes visible on their disk plane. A significant number of galaxies are edge-on or somewhere in between edge-on and face-on. Indeed HI has been detected in early type galaxies (Morganti, et al. 2006; Oosterloo, et al. 2007; Grossi, et al. 2009; Serra, et al. 2012), but these do not go beyond $M_{\mathrm{HI}} \sim 10$. Schiminovich, et al. (2010) find that the $47 \%$ of the total local SFR density is found for $M_{\text {star }}>10$ in the GASS sample. CO (a tracer for $\mathrm{H}_{2}$ and a proxy for star formation) detections have also been reported for a fraction of the GASS sample (Saintonge, et al. 2011). Given that there is little correlation between ( $u$ $r$ ) color and $M_{\text {star }}$ at these masses and the fact that GASS (and ALFALFA) detections are predominantly in the blue cloud (Catinella, et al. 2010) we would expect these red gas rich galaxies to contribute a negligible fraction to the local SFR density. This does not mean that individually all luminous red, gas rich galaxies have low star formation rates but, rather, their numbers are so small that their total contribution is negligible. A fraction of these red galaxies would then be the ones transitioning from a blue star-forming phase to a red passive phase with little star formation and another fraction will be dusty star forming galaxies, while the rest would be passively evolving. The amount of reddening would be enhanced if they are edge-on and would redden the color of disky galaxies which are either transitioning to the red phase or contain considerable dust on their disks (Graham \& Worley 2008; Tempel, et al. 2011).

Although the luminous red galaxies are the dominant population at the high mass end $\left(M_{\mathrm{HI}} \geq 10.4\right)$ the observed counts $(60 \%$ of total) are only $50 \%$ more than those of the luminous blue (40\% of total) population. When plotted on a logarithmic scale the differences between these two mass functions are not very large (see top row of figure 5). If the inclination and reddening are important and the magnitudes are corrected for them, then a fraction of gas rich red galaxies would move to the luminous blue population and would bring the mass functions of these two populations closer to each other at the high mass end. Conversely if the reddening is increased due to inclination we would expect that the effects would be more dramatic in HI velocity width function. This is indeed seen for ALFALFA galaxies (Moorman, et al. 2014), where the HI velocity width function for the red and blue are well segregated at the high velocity end. We therefore believe that reddening due to dust and inclination can partially explain why the red sample is the dominant population at the high mass end of the HIMF.

The results of section 3 are essentially conditional HIMF integrated over a range in color and magnitude, which we have called the HIMF for different populations. We can repeat the exercise and compute the HIMF in finer intervals of color and magnitude to obtain a conditional HIMF (conditioned on luminosity and color). This will then tell us about the distribution of $\mathrm{HI}$ in the color-magnitude plane. As an application one can then make better estimates of the corrections applied to $\Omega_{\mathrm{HI}}$ with the stacking methods (Rhee, et al. 2013, 2016, 2018) at higher redshifts. A second application would be to inform a proposed HI survey, which galaxy populations to stack on to make a tentative detection.

In the survey volume considered in this work we find that only $11 \%$ of the red population in SDSS have HI detections in ALFALFA. This number is $38 \%$ for blue galaxies. On the other hand $98 \%$ of ALFALFA galaxies have optical counterparts. The detections are due to a combination of total HI signal and observed HI velocity widths. The question we wish to ask is are the non-detections in the red cloud due to insufficient HI gas or due to large velocity widths or both? We argue that in the luminous red sample the nondetections are due to insufficient HI gas as well as large widths and HI masses should decrease with either increasing stellar mass or halo mass. Although figure 7 suggests that the most massive galaxies (large stellar mass, $M_{\text {star }}$ ) are also the richest in terms of their gas content, this relation is biased since it is from an HI selected sample. The appropriate sample is the GASS sample which is selected on stellar mass. We look at the $M_{\mathrm{HI}}-M_{\text {star }}$ relation in the final data release of GASS (DR5), which is summarized on table 1 of Catinella, et al. (2013). The relation has nearly a flat slope for $M_{\text {star }} \in[10.76,11.30]$, being slightly positive if all non-detections have been assigned the limiting HI mass and slightly negative if all non-detections are assigned zero HI mass. The last bin however has only a handful of ob- 


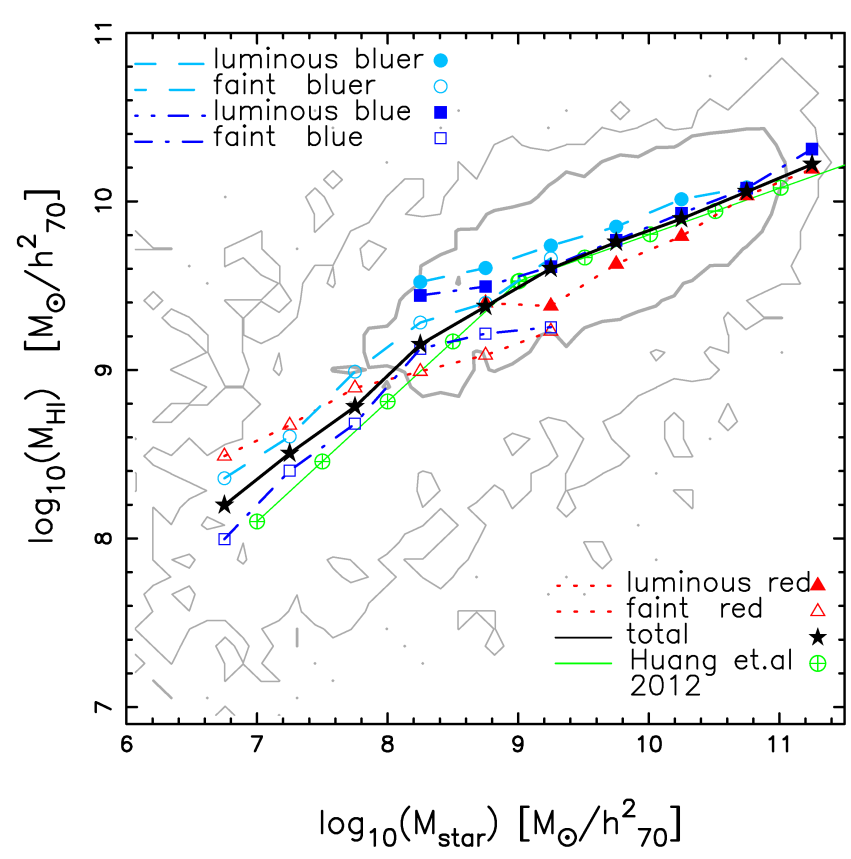

Figure 7. The $M_{\text {star }}-M_{\mathrm{HI}}$ relation for the $1.5 \sigma$ sample. The filled star (thick solid line) represents the total sample excluding the dark galaxies. The results are compared with Huang, et al. (2012) (crossed-circle and thin solid line). other data points and line styles are the same as in figure 5 .

jects which are dominated by non-detections. However the GASS and ALFALFA catalogs are relatively shallow as compared to optical surveys like the SDSS and would miss a significant number of massive galaxies. The tail of the stellar mass function is dominated by the red galaxies and at $M_{\text {star }}=11.3$ the number of red galaxies is $\sim 10 \times$ the blue galaxies (Baldry, et al. 2012). These galaxies are probably central red galaxies (Drory, et al. 2009) and would be in halos of $\log _{10}\left(M_{\text {halo }} / M_{\odot}\right) \simeq 14-14.5$ (Behroozi, Conroy \& Wechsler 2010) with virial temperatures $T_{\text {vir }} \sim 10^{7} \mathrm{~K}$ and circular velocities $V_{\text {circ }} \sim 6-9 \times 10^{2} \mathrm{~km} . \mathrm{s}^{-1}$. Most of these galaxies will then be the central galaxies of large groups of galaxies or clusters of galaxies. Given the large virial temperatures and large circular velocities it would be very unlikely to detect a considerable amount of neutral hydrogen in these systems. It is then very likely that the $M_{\mathrm{HI}}-M_{\text {star }}$ relation will not asymptote to a constant as indicated in Catinella, et al. (2013) but rather decrease with increasing stellar mass. This is suggested in the results of Kim, et al. (2017) and Spinelli, et al. (2019).

If the average $M_{\mathrm{HI}}-M_{\text {star }}$ becomes a non-monotonic function of stellar mass and therefore halo mass, HI abundance matching techniques, used to obtain $M_{\mathrm{HI}}-M_{\text {halo }}$ relation (Khandai, et al. 2011; Padmanabhan \& Kulkarni 2017), will break down. The HI HOD models which also assume a step-like function (with the help of the error function) (Guo, et al. 2017; Paul, Choudhury \& Paranjape 2018) for the average occupation of centrals, may need to be revised. A log normal form for the mean occupation function for centrals was compared to the step-like parameterization in the context of describing quasar clustering (Shen, et al. 2013), but it was found that the HOD parameters were not well constrained. Only more direct observations will shed light on the HI content of these massive galaxies and hopefully provide better inputs for the HOD parameterization.

We end this section by discussing how the sensitivity limits of ALFALFA may affect our results. We start with targeted HI observations, more sensitive than ALFALFA, that look at the HI content of massive galaxies and also luminous early type galaxies (ETGs) in the local Universe. In the GASS survey (Catinella, et al. 2010, 2013) the targets were selected by stellar mass, $10<M_{\text {star }}<11.5$ from an area common to SDSS (Abazajian, et al. 2009), GALEX (Martin, et al. 2005) and ALFALFA (Giovanelli, et al. 2005) in the redshift range $0.025<z<0.05$. The detection limit for GASS was set to a very low HI gas mass fraction of $f_{\mathrm{HI}, \lim }=M_{\mathrm{HI}, \lim } / M_{\text {star }}>0.015$ for $M_{\text {star }}>10.5$, and a constant HI gas mass limit of $M_{\mathrm{HI}}$, lim $=8.7$ for smaller stellar mass targets, which translates roughly to upper HI mass limits $8.7 \leq M_{\mathrm{HI}}$, lim $\leq 9.7$ for the non-detections. Since the maximum limiting mass for the more sensitive GASS survey, $M_{\mathrm{HI}}^{\max } \lim =9.7$, is below the characteristic mass, $M_{*}$, for the populations considered in this work (see table 1), the sensitivity limit of ALFALFA does not affect the large mass $\left(M_{\mathrm{HI}}>M_{*}\right)$ end of the HIMF.

As of the final data release from the total of 666 targeted galaxies in GASS, 287 are non detections (Catinella, et al. 2013). The non-detections span the entire targeted stellar mass range, they are mostly redder in color (NUV-r), have larger concentration index and the detection fraction is about $70 \%$ for $M_{\text {star }}<10.7$ and drops to $40 \%$ beyond that. As discussed earlier the detection fraction in ALFALFA is $11 \%(38 \%)$ for red(blue) galaxies in SDSS. Although lower than GASS, this is consistent with the trend seen in GASS where the non-detections are dominated by red galaxies. We would then expect that there is a non-negligible population of massive galaxies $M_{\text {star }}>10$, dominated by the bright red population, which could host HI gas masses upto $M_{\mathrm{HI}, \lim }=9.7$, and have not been detected by ALFALFA due to their large velocity widths. The HI mass of these objects would then be anywhere in between 0 and $M_{\mathrm{HI}}$, lim.

The ATLAS ${ }^{3 D}$ HI survey (Serra, et al. 2012) complements the results of the GASS survey by reporting HI masses of ETGs (elliptical E and lenticular S0). The ATLAS ${ }^{3 D}$ ETG sample is morphologically selected from a volume limited parent sample of 871 nearby $\left(D<42 \mathrm{Mpc},\left|\delta-29^{\circ}\right|<\right.$ $\left.35^{\circ},|\mathrm{b}|>15^{\circ}\right)$ galaxies brighter than $M_{\mathrm{K}}<-21.5$ which translates to stellar masses $M_{\text {star }} \geq 9.78$ (Cappellari, et al. 2011). Of the parent sample of 871 galaxies, 611 (70\%) are spirals, $192(22 \%)$ are lenticular and $68(8 \%)$ are elliptical. $95 \%$ of the ETGs lie on the red sequence (as demarcated by eq. 3). The ATLAS ${ }^{3 \mathrm{D}}$ HI observations are done for 166 ETGs $\left(\delta>10^{\circ}\right)$ with the Westerbork Synthesis Radio Telescope of which there are $53(32 \%)$ detections and $113(68 \%)$ non detections. The ATLAS ${ }^{3 \mathrm{D}}$ sample therefore represents massive, red, E or S0 type galaxies in the local Universe. The HI detections of ETGs have a broad distribution in the range $M_{\mathrm{HI}} \in[7,9.5]$. On the other hand the HI mass (accessed from the HyperLeda ${ }^{4}$ database) distribution of spirals in the parent sample are much narrower, having a peak at $M_{\mathrm{HI}} \sim 9.3$ and a tail at $M_{\mathrm{HI}} \sim 8$ (Serra, et al. 2012). The distribution of limiting masses of HI non-detections is in 
the range $M_{\mathrm{HI}}, \lim \in[6.5,8.5]$ peaking at $M_{\mathrm{HI}}, \lim \sim 7.1$. The HI distributions of detected ETGs and spirals overlap significantly which means that a non-negligible fraction of ETGs contain as much $\mathrm{HI}$ as in spirals. The HIMF of ETGs have a relatively low value of the characteristic mass $M_{*}=9.26$, which is a factor of 5 smaller than $M_{*}=9.96$ for the full ALFALFA population and a factor of 6 smaller than $M_{*}=10.04$ for the luminous red population of ALFALFA. The difference between HI column density distribution $\left(N_{\mathrm{HI}}\right)$ for ETGs and spirals in the ATLAS $^{3 \mathrm{D}}$ survey is significant. The characteristic column density (when the $N_{\mathrm{HI}}$ distribution is parametrized by a Schecter function) is $N *=9.2 \times 10^{19} \mathrm{~cm}^{-2}$ for ETGs and $N *=1.03 \times 10^{21} \mathrm{~cm}^{-2}$. Therefore the HI in gas rich ETGs is rarely as dense as the average column densities of spirals (Serra, et al. 2012). Given that the GASS and the ATLAS ${ }^{3 \mathrm{D}}$ HI surveys are targeted surveys, more sensitive than ALFALFA, and are specifically looking at the HI gas content of massive galaxies we conclude that it is very unlikely that ALFALFA has missed out any galaxy with masses $M_{\mathrm{HI}}>10$ due to an unusually large velocity width. However due to their moderate amounts of HI individual detections are less frequent in ALFALFA as compared to these surveys.

Finally it is worth considering how a different choice of the sensitivity limit (e.g. dictated by high $\mathrm{S} / \mathrm{N}$ objects Code 1 objects in this work and in Martin, et al. 2010) of ALFALFA in turn affects the HIMF. For example, could a lower sensitivity limit, by including lower S/N Code 2 objects, alter the HIMF? Haynes, et al. (2011) addressed this question by considering Code 1 and 2 sources, which in turn lowers the sensitivity limit compared to Code 1 only objects (see eqs. 6 and 7 and discussion in section 6 of Haynes, et al. 2011). Both the amplitude and characteristic mass of HIMF remained unchanged, however the faint-end slope of the HIMF decreased from $\alpha=-1.33 \pm 0.02$ (Code 1) to $\alpha=-1.29 \pm 0.02$ (Code 1 and 2). They concluded that there is little value in adding lower $\mathrm{S} / \mathrm{N}$ objects in the analysis and that statistical estimators like the 2DSWML method are robust to such changes.

In a separate analysis Papastergis, et al. (2012) explored if there were systematic differences in estimating the HIMF by considering an HI-selected sample and an optically-selected sample. The HI-selected sample is the $\alpha .40$ sample and the corresponding estimate of the HIMF is found with methods similar to the one described in this work. The optically-selected sample consists of all the HI detections and non-detections. The non-detections are the SDSS galaxies in the same volume as ALFALFA which do not have HI detections. These galaxies are assigned a lower and upper limiting $\mathrm{HI}$ mass $\left(M_{\mathrm{HI}, \lim }^{\min }, M_{\mathrm{HI}, \lim }^{\max }\right)$. The lower limit is $M_{\mathrm{HI}, \lim }^{\mathrm{min}}=0$. The upper limit is computed by converting the detection limit, which is the $25 \%$ completeness limit (eqs. 5 and 7 in Haynes, et al. 2011), to an HI mass. To estimate $M_{\mathrm{HI}}^{\max }$ lim we need a distance (which exists) and velocity width, $W_{50}$ (which has to be estimated). Papastergis, et al. (2012) used the average stellar mass Tully-Fisher relation, $M_{\text {star }}-V_{\text {rot }}$ for the $\alpha .40$ galaxies to estimate $W_{50}$ for the non-detections, after accounting for inclination effects. The optically-selected sample then consists of ALFALFA detections and two estimates of HI masses for the non-detections. The optically-selected sample is an r-band flux limited sample which has a different selection function as compared to the HI-selected sample. The HIMF estimated from the optically-selected and HI-selected sample should broadly be consistent with each other. A naive expectation is that the HIMF from the HI selected-sample should lie in between the two estimates of the HIMF from the optically-selected sample. In the limit that the assigned lower and upper limiting HI masses approach the true HI mass of the undetected source we expect the HIMF estimated from the opticallyselected sample to approach that of the HI-selected sample. We also point out at the high mass end, all the estimates of the HIMF should be the same. This is demonstrated in figure 6 of Papastergis, et al. (2012). Given the uncertainties associated in obtaining $M_{\mathrm{HI}, \lim }^{\max }$ which use average scaling relations and inclination effects (which are prone to errors) we do not comment further on the differences between the HIMF from the two samples. Based on the arguments presented above, we believe that although the sensitivity of ALFALFA affects the individual detections as compared to more sensitive targeted surveys, the estimates of the HIMF for different populations considered here are robust.

\section{SUMMARY}

In this work we have measured the HIMF of different galaxy populations picked from the color-magnitude plane. The galaxies considered were from a local volume common to ALFALFA and the SDSS surveys in the redshift range $z \in[0,0.05]$. After putting the relevant cuts in quality of detection, volume and completeness the final sample analyzed consists of 7857 galaxies. We divided the total sample first into luminous and faint populations (cut in magnitude) and these were further split into three colors: red, blue and bluer. This forms a disjoint set of six populations. A seventh population which we call a dark population is one which does not have any optical counterparts in SDSS but has a detection in ALFALFA. The union of these seven populations is the total sample of 7857 galaxies. We have further considered a second sample which redefines the six populations by shifting the boundaries defining them. We have called them the $1 \sigma$ and $1.5 \sigma$ samples (section 2.1). The reason for doing this is to illustrate that our sample definition does not change the qualitative results that we report. The reason for splitting the full blue cloud into four (faint/luminous for blue/bluer) instead of just two (faint/luminous for all blue) was because we wanted to see the systematic effect of the tail of the blue population (especially the faint end) on our results. We indeed find that based on how we define our sample the faint bluer population becomes the dominant population at the low mass end of the HIMF (figure 5 and tables $1 \& 2$ ).

We summarize our results below:

- For a fixed range in color, in the color-magnitude plane (e.g. red, blue or bluer), the HIMF of the luminous population dominates over their corresponding faint counterparts at the knee and the large mass end whereas the faint populations dominate at the low mass end.

- For a fixed magnitude interval, in the color-magnitude plane (e.g. luminous or faint) there is no systematic trend at the low mass end and the knee of the HIMF, with decreasing color, for the faint population. However for the luminous population, we see that the HIMF at the high mass end 
increases with increasing color. The luminous red population is the dominant population at this end.

- The luminous red population dominates the total HIMF at $M_{\mathrm{HI}} \geq 10.4$. When combined with the faint red sample it contributes about $\sim 16-17 \%$ of the $\Omega_{\mathrm{HI}}$ budget. The dark population contributes $\sim 3 \%$ to $\Omega_{\mathrm{HI}}$. This has implications for detections done with stacking at higher redshifts which would target the blue star forming cloud for a first detection.

- The total blue cloud (blue and bluer) represents about $\sim 80 \%$ of the $\Omega_{\mathrm{HI}}$ budget.

- In the mass range about the knee, $M_{\mathrm{HI}} \in[8,10.4]$, the dominant populations are the faint and luminous blue populations with the latter dominating at larger masses in this interval. Their total contribution to $\Omega_{\mathrm{HI}}$ is $\sim 55-70 \%$ depending on sample definitions.

- The dominant populations contributing to the low mass end of the HIMF are the faint blue and faint bluer populations, the latter being dominant only for the $1 \sigma$ sample definition.

- The luminous bluer and faint red populations are subdominant populations contributing a total of $\sim 6-10 \%$ to $\Omega_{\mathrm{HI}}$. For the $1.5 \sigma$ sample the fraction of luminous bluer(faint red) galaxies in ALFALFA to that in SDSS is $41 \%$ (28\%). In comparison the detection rate of ALFALFA in the red cloud is $11 \%$ and the blue cloud is $38 \%$. This shows that although the detection rate of ALFALFA for the faint red population is higher than the luminous red population their contribution to $\Omega_{\mathrm{HI}}$ is small. This is because the number density of these galaxies is small and the HI mass of these galaxies is also small. On the contrary the luminous blue galaxies are HI rich (figure 7) on average but due to their small number densities (figure 5) they too contribute little to the $\Omega_{\mathrm{HI}}$ budget.

It would be interesting to see if simulations (Davé, et al. 2017), SAMs (Kim, et al. 2017) and HOD models (Paul, Choudhury \& Paranjape 2018) are able to reproduce our results which provide additional constraints on the population of HI selected galaxies. In section 4 we argued that the effect of dust and inclination are responsible for the luminous red population dominating the total HIMF at large masses. This was based on the results of Catinella, et al. (2013); Moorman, et al. (2014) but needs to be confirmed with a more detailed followup. In a forthcoming paper we will report on a more detailed analysis of the HI velocity width function. We are also working on measuring the conditional (conditioned on color or magnitude or both) HIMF which will put additional constraints on the properties of gas bearing galaxies.

\section{ACKNOWLEDGMENTS}

We would like to thank R. Srianand and A. Paranjape for the many useful discussions that we had throughout this work. SD would like to thank R. Srianand for giving the hands-on training on obtaining and processing SDSS data. We would like to acknowledge discussions with J. S. Bagla and N. Kanekar. The research of NK is supported by the Ramanujan Fellowship awarded by the Department of Science and Technology, Government of India. All the analyses were done on the xanadu server funded by the Ramanujan Fellowship. NK acknowledges the support from the In- ter University Centre for Astronomy and Astrophysics (IUCAA) associateship programme. We would like to thank the referee for useful comments which helped in improving the presentation of this work.

We would like to acknowledge the work of the entire ALFALFA collaboration in observing, flagging, and extracting the properties of galaxies that this paper makes use of. This work also uses data from SDSS DR7. Funding for the SDSS and SDSS-II has been provided by the Alfred P. Sloan Foundation, the Participating Institutions, the National Science Foundation, the U.S. Department of Energy, the National Aeronautics and Space Administration, the Japanese Monbukagakusho, the Max Planck Society, and the Higher Education Funding Council for England. The SDSS Website is http://www.sdss.org/. The SDSS is managed by the Astrophysical Research Consortium for the Participating Institutions.

\section{REFERENCES}

Abazajian, K. N., et al. 2009,ApJS,182, 543

Baldry, I. K., et al. 2004, ApJ,600,681

Baldry et al., 2012, MNRAS, 421, 621

Behroozi P. S., Conroy C., Wechsler R. H., 2010, ApJ, 717, 379

Bigiel F., et al., 2008, AJ, 136, 2846

Blanton M. R., Roweis S., 2007, AJ, 133, 734

Bruzual G., Charlot S., 2003, MNRAS, 344, 1000

Cannon, J.M., et al., 2015, Astro J 149, 72

Cappellari M., et al., 2011, MNRAS, 413, 813

Casey C. M., Narayanan D., Cooray A., 2014, PhR, 541, 45

Catinella B., et al., 2010, MNRAS, 403, 683

Catinella B., et al., 2013, MNRAS, 436, 34

Chang T.-C., Pen U.-L., Bandura K., Peterson J. B., 2010, Natur, 466, 463

Davé R., Rafieferantsoa M. H., Thompson R. J., Hopkins P. F., 2017, MNRAS, 467, 115

Davis M., Huchra J., 1982, ApJ, 254, 437

Di Matteo T., Springel V., Hernquist L., 2005, Nature, 433, 604

Drory N., et al., 2009, ApJ, 707, 1595

Efstathiou G., Ellis R. S., Peterson B. A., 1988, MNRAS, 232,431

Giovanelli R., et al., 2005, AJ, 130, 2598

Graham A. W., Worley C. C., 2008, MNRAS, 388, 1708

Grossi M., et al., 2009, A\&A, 498, 407

Guo, H., et al.,2017, AJ 846, Issue 1, article id. 61, pp. 23

Haynes M. P., et al., 2011, AJ, 142, 170

Haynes M. P., et al., 2018 ApJ 861, 49

Huang S., Haynes M. P., Giovanelli R., Brinchmann J., 2012, ApJ, 756, 113

Janowieki, S., et al., 2015, Ap.J. 801, 96

Jones, M.G., Haynes, M.P., Giovanelli, R. \& Moorman, C. 2018 MNRAS 477, 2

Kanekar N., Sethi S., Dwarakanath K. S., 2016, ApJL, 818, L28

Kennicutt R. C., 1989, ApJ, 344, 685

Kennicutt R. C., 1998, ApJ, 498, 541

Kereš D., Katz N., Fardal M., Davé R., Weinberg D. H., 2009, MNRAS, 395, 160 
Khandai N., et al., 2011, MNRAS, 415, 2580

Kim H.-S. et al., 2017, MNRAS, 465, 111

Lah P., et al., 2007, MNRAS, 376, 1357

Lah P., et al., 2009, MNRAS, 399, 1447

Le Floc'h E., et al., 2005, ApJ, 632, 169

Leisman, L., et al., 2017, Ap J 842, 133

Leroy A. K. et al., 2008, AJ, 136, 2782

Loveday, J., 2000, MNRAS,312,557

Madau P., Dickinson M., 2014, ARA\&A, 52, 415

Maddox N., Hess K. M., Obreschkow D., Jarvis M. J., Blyth

S.-L., 2015, MNRAS, 447, 1610

Martin D. C., et al., 2005, ApJL, 619, L1

Martin, A., et al., 2010, ApJ, 723,1359

Martin A. M., Giovanelli R., Haynes M. P., Guzzo L., 2012, ApJ, 750, 38

Masters, K.L. 2005, PhD Thesis, Cornell University

Masui K. W., et al., 2013, ApJL, 763, L20

Meyer M. J., et al., 2004, MNRAS, 350, 1195

Moorman C. M., et al., 2014, MNRAS, 444, 3559

Morganti R., et al., 2006, MNRAS, 371, 157

Neeleman M., et al., 2016, ApJ, 818, 113

Noterdaeme P., et al., 2012, A\&A, 547, L1

Oosterloo T. A., Morganti R., Sadler E. M., van der Hulst

T., Serra P., 2007, A\&A, 465, 787

Padmanabhan H., Kulkarni G., 2017, MNRAS, 470, 340

Papastergis, A., Cattaneo, A., Huang, S., Giovanelli, R. \&

Haynes, M.P. 2012, ApJ 759, 138

Papastergis E., Giovanelli R., Haynes M. P., Rodríguez-

Puebla A., Jones M. G., 2013, ApJ, 776, 43

Parkash V., Brown M. J. I., Jarrett T. H., Bonne N. J., 2018, ApJ, 864, 40

Paul N., Choudhury T. R., Paranjape A., 2018, MNRAS, 479, 1627

Prochaska J. X., Herbert-Fort S., Wolfe A. M., 2005, ApJ, 635, 123

Rao S. M., Turnshek D. A., Sardane G. M., Monier E. M., 2017, MNRAS, 471, 3428

Rhee J., et al., 2013, MNRAS, 435, 2693

Rhee J., Lah P., Chengalur J. N., Briggs F. H., Colless M., 2016, MNRAS, 460, 2675

Rhee J., et al., 2018, MNRAS, 473, 1879

Said K., Kraan-Korteweg R. C., Staveley-Smith L., 2019, MNRAS, 486, 1796

Saintonge A., et al., 2011, MNRAS, 415, 32

Sandage A., Tammann G. A., Yahil A., 1979, ApJ, 232, 352

Schiminovich D., et al., 2010, MNRAS, 408, 919

Schlegel, David J.; Finkbeiner, Douglas P.; Davis, Marc, 1998, ApJ, 500, 525

Schmidt M., 1959, ApJ, 129, 243

Schmidt M., 1963, ApJ, 137, 758

Schmidt, M., 1968, ApJ,151,393

Serra P., et al., 2012, MNRAS, 422, 1835

Shen Y., et al., 2013, ApJ, 778, 98

Spinelli M., Zoldan A., De Lucia G., Xie L., Viel M., 2019, arXiv, arXiv:1909.02242

Springel V., Hernquist L., 2003, MNRAS, 339, 289

Suess, K.A., Darling, J., Haynes, M.P. \& Giovanelli, R., 2016, MNRAS 459, 220

Tempel E., et al., 2011, A\&A, 529, A53

Tramonte D., Ma Y.-Z., Li Y.-C., Staveley-Smith L., 2019, MNRAS, 489, 385
Willmer C. N. A., 1997, AJ, 114, 898

Zwaan M. A., et al., 2003, AJ, 125, 2842

Zwaan M. A., Meyer M. J., Staveley-Smith L., Webster R. L., 2005, MNRAS, 359, L30

Zwaan M. A., Meyer M. J., Staveley-Smith L., 2010, MNRAS, 403, 1969

\section{APPENDIX A: THE 2DSWML METHOD TO CALCULATE THE HI MASS FUNCTION}

We now proceed in describing our implementation of the 2DSWML method (Zwaan, et al. 2003; Martin, et al. 2010). Assuming a bivariate distribution $\phi\left(M_{\mathrm{HI}}, W_{50}\right)$ the probability of detecting a galaxy ' $i$ ' with HI mass $M_{\mathrm{HI}}^{i}$ and profile width $W_{50}^{i}$ at distance $D^{i}$ is

$p_{i}=\frac{\phi\left(M_{\mathrm{HI}}^{i}, W_{50}^{i}\right)}{\int_{W_{50}=0}^{\infty} \int_{M_{\mathrm{HI}}=M_{\mathrm{HI}, l i m}\left(D^{i}, W_{50}^{i}\right)}^{\infty} \phi\left(M_{\mathrm{HI}}, W_{50}\right) d M_{\mathrm{HI}} d W_{50}}$

We now need to discretize the above equation for the 2DSWML method. We will begin by considering the distribution of galaxies in bins of $M=\log _{10}\left[M_{\mathrm{HI}} / M_{\odot}\right]$ and $W=$ $\log _{10}\left[W_{50} /\left(\mathrm{km}_{\mathrm{s}} \mathrm{s}^{-1}\right)\right]$. The number of bins are $N_{M} \& N_{W}$ and the bin widths are $\Delta M \& \Delta W$ respectively. Therefore the two dimensional distribution can be parameterized by $\phi_{j k}$, where $j=0,1,2, \ldots, N_{M}-1$ and $k=0,1,2, \ldots, N_{W}-1$. In this analysis we have taken 10 bins per dex in velocity width and 5 bins per dex in mass. Eq A1 for the 2DSWML is now

$$
p_{i}=\frac{\Sigma_{j} \Sigma_{k} V_{i j k} \phi_{j k}}{\Sigma_{j} \Sigma_{k} H_{i j k} \phi_{j k} \Delta M \Delta W}
$$

Here $V_{i j k}$ ensures that galaxy ' $i$ ' is only populated in its corresponding ' $j-k$ ' bin.

$V_{i j k}= \begin{cases}1, & \text { if galaxy } i \text { is a member of mass bin } j \\ \text { and profile width bin } k . & \text { otherwise. }\end{cases}$

$H_{i j k}$ is a weight corresponding to galaxy ' $i$ ' in the ' $j-k$ ' bin and takes values from 0 to 1 . It appears so that the integral in the denominator of eq. A1 can be done in the entire $M-W$ plane after convolving with the completeness function $C^{i}$ in the $M-W$ plane. An example for one of the galaxies in our sample is shown in figure A1. The solid broken line is the completeness relation for this object in the $M$ - $W$ plane. The shaded(white) area is the area accessible(inaccessible) to this object. The shaded area is given by:

$$
\begin{aligned}
& \Delta M \Delta W \sum_{k=0}^{N_{W}-1} \sum_{j=0}^{N_{M}-1} H_{i j k}= \\
& \int_{W=W_{0}}^{W=W_{N_{W}-1}} \int_{M=M_{0}}^{M=M_{N_{M}-1}} C^{i}(M, W) \mathrm{d} M \mathrm{~d} W
\end{aligned}
$$

Computing $H_{i j k}$ is a straightforward exercise and we have shown it as diagrams in table A1. The shaded area (column 2) in each case is the $j$ - $k$ bin of interest for which $H_{i j k}$ has to be computed. In cases 1 and 2 , the values of $H_{i j k}$ are either 1 or 0 . This is because the completeness line does not intersect the square and lies below or above that 
$[\mathrm{h}]$

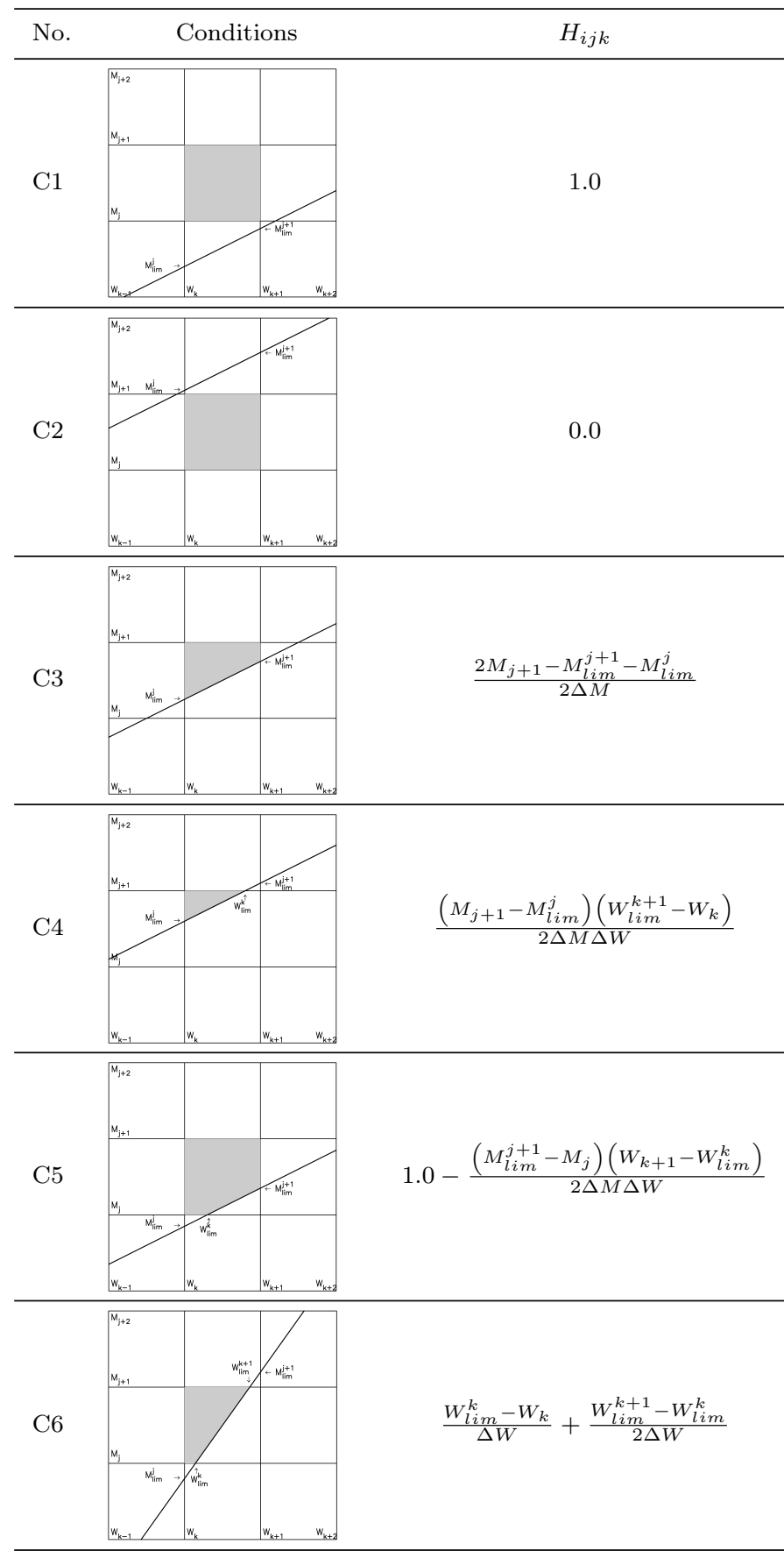

Table A1. Values of $H_{i j k}$ (column 3) are shown diagrammatically (column 2). The shaded region corresponds to the $j$ - $k$ bin of interest. Cases 1 and 2 (first two rows) take values 1 and 0 , the completeness curve (line) lies below or above the square and never intersects it. Cases 3-6 give fractional values of $H_{i j k}$ since the $j$ $k$ bin of interest intersects the completeness curve. The points of intersection are denoted by $W_{\mathrm{lim}}^{j}, M_{\mathrm{lim}}^{k}$ if it does not exactly intersect on the bin edges $W_{k}, M_{j}$.

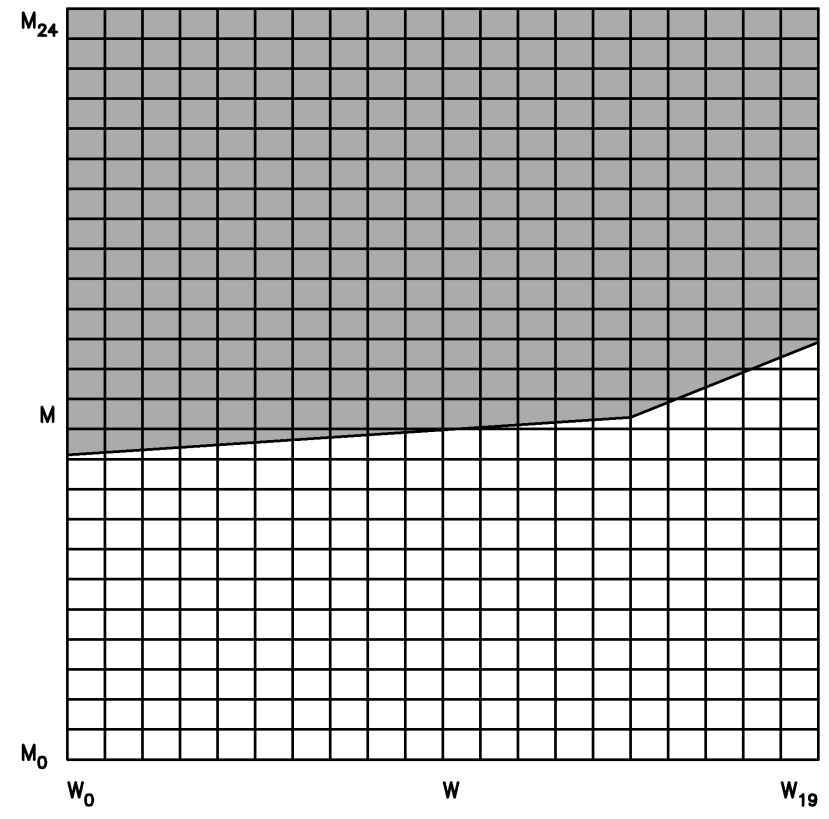

Figure A1. The completeness relation (broken solid line) is shown in the M-W plane. This particular object ' $i$ ' has the following properties: $\log _{10}\left[M_{\mathrm{HI}}^{i} / M_{\odot}\right]=8.73,\left[W_{50}^{i} / \mathrm{km} \cdot \mathrm{s}^{-1}\right]=$ $93,[D / \mathrm{Mpc}=27]$. The shaded(white) region is the area accessible(inaccessible) to this object in the $M-W$ plane.

particular bin of interest. In cases 3-5 the square of interest intersects with the completeness curve and the shaded area is the value that $H_{i j k}$ takes (column 3 ) which are fractional values. The points of intersection are denoted as $W_{\mathrm{lim}}^{k}, M_{\mathrm{lim}}^{j}$ if it does not exactly intersect on the bin edges $W_{k}, M_{j}$. Case 6 is introduced and assumes a completeness slope greater than 1, which is not the case for ALFALFA. We also note that the completeness relation eq. 2 has a change in slope at

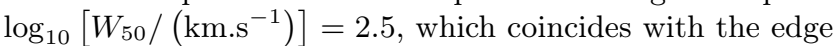
of the bin in $W_{50}$ for our choice. Table A1 assumes this so that no further cases are considered.

In the 2DSWML one wishes to find $\phi_{j k}$ that maximizes the joint probability or likelihood of finding all the galaxies in the sample simultaneously

$\mathcal{L}=\prod_{i=1}^{N_{g}} p_{i}$

Using eq. A1 the joint likelihood is

$\mathcal{L}=\prod_{i=1}^{N_{g}} \frac{\Sigma_{j} \Sigma_{k} V_{i j k} \phi_{j k}}{\Sigma_{j} \Sigma_{k} H_{i j k} \phi_{j k} \Delta m \Delta w}$

To obtain $\phi_{j k}$, we maximize rather the log-likelihood

$\ln \mathcal{L}=\sum_{i=1}^{N_{g}} \ln \left(\frac{\Sigma_{j} \Sigma_{k} V_{i j k} \phi_{j k}}{\Sigma_{j} \Sigma_{k} H_{i j k} \phi_{j k} \Delta m \Delta w}\right)$

This gives us

$$
\begin{aligned}
\phi_{j k} & =\left[\Sigma_{i} V_{i j k}\right]\left[\Sigma_{i} \frac{H_{i j k}}{\Sigma_{m} \Sigma_{n} H_{i m n} \phi_{m n}}\right]^{-1} \\
& =n_{j k}\left[\Sigma_{i} \frac{H_{i j k}}{\Sigma_{m} \Sigma_{n} H_{i m n} \phi_{m n}}\right]^{-1}
\end{aligned}
$$

where, $n_{j k}=\sum_{i} V_{i j k}$ is the number of galaxies in mass bin $j$ 
and profile width bin $k . \phi_{j k}$ are iteratively determined from eq. A8. To start the iteration we set the initial value of on the RHS of eq. A8 to be $\phi_{m n}^{i n}=n_{j k} /\left[V_{\text {surv }} \Delta M \Delta W\right]$. We set a relative tolerance for convergence of $10^{-3}$ for all $\phi_{j k}$. We find that $\phi_{j k}$ converges within 20 iterations. Finally the HIMF is obtained by summing over the velocity width bins $k$.

$\phi_{j}=\sum_{k} \phi_{j k} \Delta w$

\section{A1 Normalization of HIMF}

One drawback for likelihood methods, as opposed to the $1 / V_{\max }$ method, is that the normalization of the HIMF is not fixed. This is obvious from eq. A1. There are a number of ways to fix the normalization (Davis \& Huchra 1982; Willmer 1997; Zwaan, et al. 2003; Martin, et al. 2010) which involve computing the selection function. Here we try a slightly different method. We assume that the high mass end of the HIMF is not affected by the selection function. One can test this assumption by comparing the ratio of the normalized mass function from 2DSWML to that of the observed mass function which is related to the observed counts. In the top panel of figure A2 the observed HIMF is shown as filled pentagons (solid line) and the un-normalized and converged HIMF from the 2DSWML is shown as filled triangles (dashed line). This is done for the $\alpha .100$ sample (Haynes, et al. 2018). The ratio of these is shown for the last 7 mass bins in the lower panel of figure A2. If the selection function affects the high mass end, the ratio at this end would not have a flat feature. We indeed find that in this particular example of $\alpha .100$ the last 3 mass bins are unaffected at the level ranging from $0-0.003 \%$; whereas the last but third bin is relatively suppressed by around $0.4 \%$. For this method to work we need to test the flatness of this ratio which means that at least the last two points at the high mass end should be unaffected by the selection function. Starting from the high mass end we progress sequentially to smaller bins which are unaffected by the selection function. The search is stopped when the selection function affects the particular bin at the level of $0.1 \%$ or greater. Finally we fix the normalization by matching the integrated counts over these bins to that of the observed HIMF.

We have compared this method to the one which normalizes the mass function to match the average observed counts as in Davis \& Huchra (1982); Martin, et al. (2010). We find that they match at the relative level of $\sim 0.4 \%$. Finally we compare in figure A3 our result with that of $\alpha .100$ HIMF (Jones, et al. 2018). As we can see our implementation with some minor modifications reproduces the HIMF of (Haynes, et al. 2018) extremely well.

\section{A2 Error Analysis of HIMF}

(i) Mass Errors: Since $M_{\mathrm{HI}} \propto S_{21} D^{2}$ the uncertainties on both integrated flux and distances lead to uncertainties on mass. Peculiar velocities of galaxies can affect distance estimates. This effect is larger in the local volume and smaller at higher redshifts where the Hubble flow dominates over peculiar velocities. The $\alpha .40$ catalog also includes radial distances (Haynes, et al. 2011), using a lo-

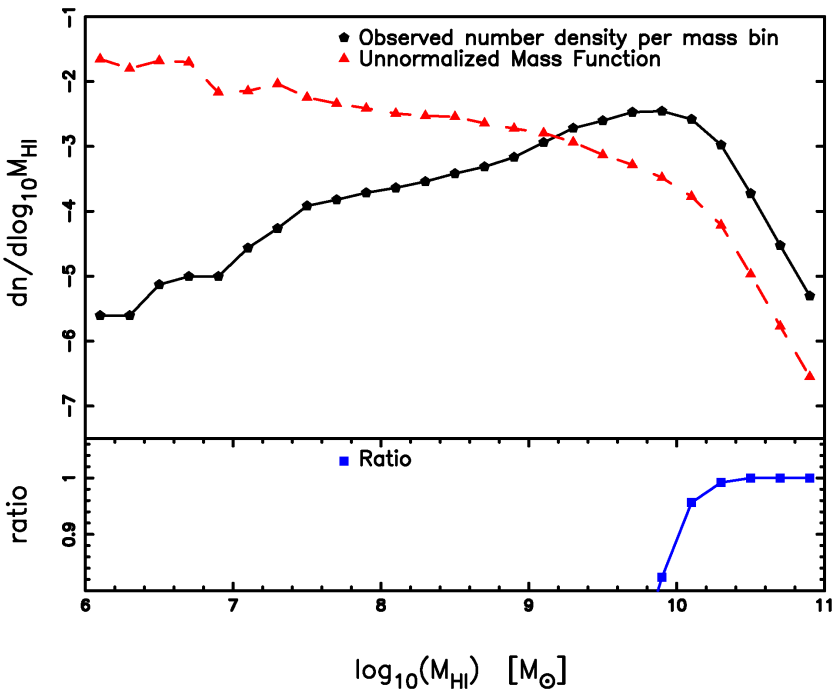

Figure A2. Upper panel: Red dashed line is the mass function estimated using 2DSWML method, which is not normalized. The black solid line is the number density per mass bin calculated for the same sample of HI galaxies. Lower panel: The blue solid line shows the ratio of the un-normalized mass function and the number density, multiplied by 100 .

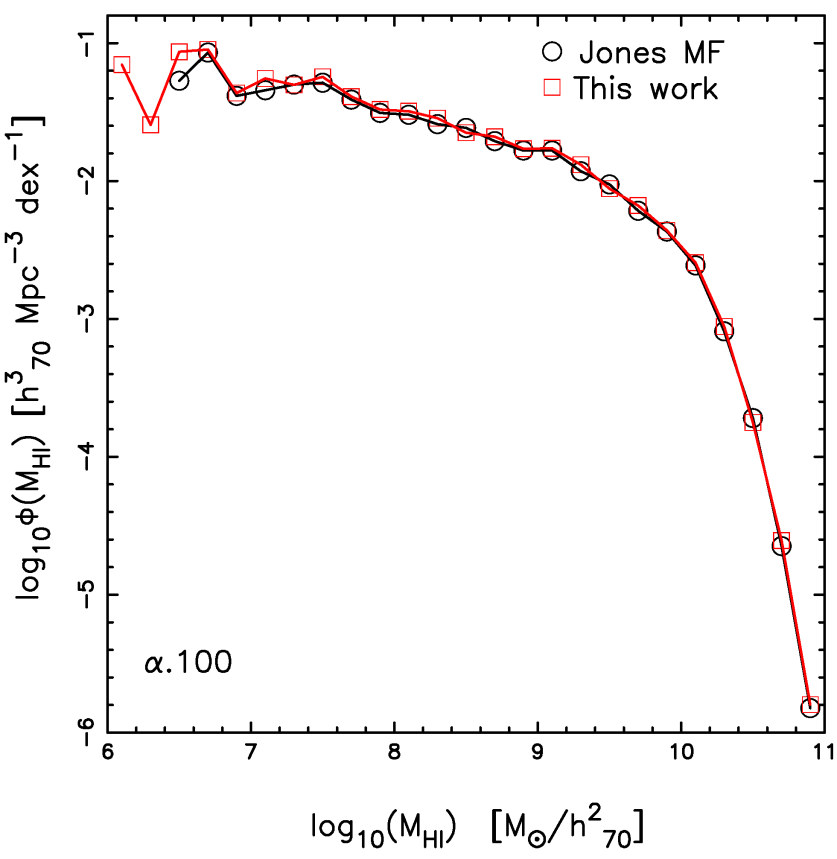

Figure A3. Comparison of the HIMF in the $\alpha .100$ sample. The open circles is the HIMF by Jones, et al. (2018). The open squares is the HIMF estimated by our implementation of the 2DSWML method.

cal volume flow model (Masters 2005) for galaxies with $c z_{\mathrm{CMB}}<6000 \mathrm{~km} . \mathrm{s}^{-1}$. This model has an estimated local velocity dispersion of $\sigma_{v}=163 \mathrm{~km} . \mathrm{s}^{-1}$. For these galaxies we take the error in the distance to be the maximum of $\sigma_{v}$ and $10 \%$ of the distance. For galaxies $c z_{\mathrm{CMB}}>6000 \mathrm{~km} . \mathrm{s}^{-1}$ distances are estimated using the Hubble and we take the error on distances to be $10 \%$. Using the errors on distance and fluxes and their observed values we generate 300 Gaus- 
sian realizations and recompute $M_{\mathrm{HI}}$ for every object. We apply the 2DSWML method to all the realizations and find out the width of the distribution for every $\phi_{j}$ which we quote as an error.

(ii)Poisson Errors: The observed count in some of the bins is very low which means that it is important to consider errors due to Poisson counting.

(iii)Sample Variance: We estimate this error by splitting the area into 26 contiguous regions of approximately equal area each. We compute the HIMF by removing one region at a time. Finally the jackknife uncertainty for $\phi_{j}$ is computed as $\sigma_{\phi^{j}}=\frac{N-1}{N} \sum_{i=1}^{N=26}\left(\bar{\phi}^{j}-\phi_{i}^{j}\right)^{2}$ where $\bar{\phi}^{j}$ is the jackknife mean and $\phi_{i}^{j}$ is the value for the $\mathrm{i}^{\text {th }}$ jackknife sample.

(iv) Other Errors: There are many other sources of errors which are discussed in Jones, et al. (2018). E.g. the error associated with the 2DSWML method which one can either estimate using the information matrix (Efstathiou, Ellis, \& Peterson 1988) or by making further mocks (Jones, et al. 2018). One needs to understand how these errors are correlated with a finite sample or Poisson errors. We also expect Poisson errors to be correlated to mass errors especially in the lowest and highest mass bins where the observed counts are low. Another source of uncertainty is the error in the velocity width $W_{50}$. Since the peak flux, $S_{\text {peak }}$, is inversely proportional to $W_{50}$ we expect their errors to be correlated. Since we integrate over $W_{50}$ to obtain the HIMF, we do not consider errors in $W_{50}$. In order to properly account for errors one will need their covariances. We have attempted to add further sources of errors in quadrature but we find that the error bars become progressively larger and the Schechter function fits have a $\chi_{\text {red }}^{2}<1$ which means that we may be overestimating the errors. For this work we stick to the errors $(i)-($ iii $)$ and add them in quadrature. These errors are comparable to Martin, et al. (2010); Haynes, et al. (2011). 